Portland State University

PDXScholar

$12-8-2020$

\title{
Vessel Trajectory Prediction Using Historical AIS \\ Data
}

Jagir Laxmichand Charla

Portland State University

Follow this and additional works at: https://pdxscholar.library.pdx.edu/open_access_etds

Part of the Electrical and Computer Engineering Commons Let us know how access to this document benefits you.

Recommended Citation

Charla, Jagir Laxmichand, "Vessel Trajectory Prediction Using Historical AIS Data" (2020). Dissertations and Theses. Paper 5628.

https://doi.org/10.15760/etd.7500

This Thesis is brought to you for free and open access. It has been accepted for inclusion in Dissertations and Theses by an authorized administrator of PDXScholar. Please contact us if we can make this document more accessible: pdxscholar@pdx.edu. 
Vessel Trajectory Prediction Using Historical AIS Data

by

\title{
Jagir Charla
}

A thesis submitted in partial fulfillment of the requirements for the degree of

\author{
Master of Science \\ in \\ Electrical and Computer Engineering
}

Thesis Committee:

John Lipor, Chair

Martin Siderius

Christof Teuscher

Portland State University

2020 


\begin{abstract}
Maritime vessel position coordinates are important information for maritime situational planning and organization. A better estimate of future locations of the maritime vessels, from their current locations, can help maritime authorities to make planned decisions, which can be helpful to avoid traffic congestion and longer waiting times. This thesis develops a method for estimating future locations of the vessels using their current and previous locations and other data.

The motivating scenario for this work is that of determining the future locations of the vessels based on their current location and previous locations for accurate modelling of underwater acoustic noise. As ambient noise levels can be reliably calculated based on ship traffic, the location of ships have a significant impact on noise level estimates. Methods to predict future locations of the vessels can be classified into two types, one which tries to model the physical behaviour of the ship, and methods which are data driven. The problem with the first approach is that there is a large number of parameters such as ocean current, weather, wind speed, vessel speed, and direction to develop an accurate physical model of the ship. Incorporating all the parameters in the physical model becomes intractable. The advantage of a data driven approach is that all the parameters, which may decide the physical behaviour of the vessel, are incorporated through the data.

We have developed two methods leveraging historical Automatic Identification System (AIS) data to come up with a model to predict the future location of the vessels. The first method uses a simple mathematical construct called the Markov model for the prediction task, which computes the transition probability matrix for the given Region of Interest (ROI), then the computed transition probability matrix is used to make predictions. The second method uses a neural network-based technique called Long-Short Term Memory (LSTM), which incorporates side information along
\end{abstract}


with location data to model the behaviour of the vessel.

We evaluated the performance of both the methods in multiple regions near the US coasts. We show empirically that the Markov model-based method, for a smaller region $\left(22,500 \mathrm{~km}^{2}\right)$, is able to predict the location of the vessel with an average error of $11.7 \mathrm{~km}$ at an interval of 4 hours. We show empirically that the LSTM-based method, which is more general and applicable for the bigger region as well, is able to predict the location of the vessels with an average error of $12.18 \mathrm{~km}$ at an interval of 4 hours. The ability of LSTM-based method to incorporate other information makes it well suited for the task of vessel trajectory prediction.

Considering future work, we propose a combination of two methods, as well as other data sources like weather and complex neural network models that may be worth further investigation. This thesis's contributions are detailed investigation and analysis of the Markov model approach and LSTM-based approach on different regions near the US coastal area. We provide a detailed analysis of the effect of adding different features to LSTM-based model. Additionally, we contribute a method for trajectory prediction for multiple timestamps in the future, which is simple and has not been tried in the current state of the art to the best of our knowledge. 
To HS. 


\section{Acknowledgements}

I would like to start by thanking my advisor, John Lipor, for his guidance and patience over the past two years. I feel truly fortunate to have had the opportunity to learn, forget, and re-learn in an environment of academic freedom and support, where showing up to every meeting with more questions than answers was met with enthusiasm and encouragement. I am grateful to be able to count John as an impactful teacher, someone I would always look up to in my life.

I want to extend my gratitude to every professor I have had the chance to learn from; each has taught me valuable lessons and shaped the student and person I am. A special thanks to Amit Goradia and Ganesh Gore for igniting my curiosity about engineering and programming, and to Amey Naik for cultivating my interest in signal processing. Thanks to Martin Siderius for guiding and supporting me in this project, and Ted Willke to show me the power of deep learning. And thanks to Christof Teuscher for saving my committee.

I would also like to thank a handful of my peers for being exceptional collaborators, mentors, supporters, and friends. To Lokesh Ramina and Sagar Dhruv for teaching me patience and persistence when answers do not come easily. To Rushabh Loladia and Hitesh Prajapati for their willingness to help me re-think and re-work anything and everything, and for instilling in me a desire to "actually" learn.

Lastly, I would like to thank my family. To my parents for their unfailing support and the incredible opportunities and experiences their hard work and love have afforded me. To my brother and sister in law, Azad and Meenal, for their relentless confidence

in me. And finally, to my friends at Goose Hollow, for helping and supporting me in the last two years. 


\section{Table of Contents}

Abstract i i

Dedication iii

Acknowledgements iv

List of Tables $\quad$ vi

List of Figures $\quad$ vii

1 Introduction 1

1.1 Motivation . . . . . . . . . . . . . . . . . . . . . . . . . 1

1.2 Current State of The Art . . . . . . . . . . . . . . . . . . . . . . . . . 2

1.3 Contributions . . . . . . . . . . . . . . . . . 5

1.3.1 Markov Model . . . . . . . . . . . . . . . . 5

1.3 .2 Neural Network-Based Model . . . . . . . . . . . . . . . 5

2 Data Pre-processing $\quad 6$

2.1 AIS Dataset . . . . . . . . . . . . . . . . . . . . . . . . . 6

2.2 AIS Message . . . . . . . . . . . . . . . . . . . . . . . . . 6

2.3 Data Pre-Processing . . . . . . . . . . . . . . . . . . . . . . . . . . . 8

\begin{tabular}{lll}
3 & Markov Model & 12 \\
\hline
\end{tabular}

3.1 Introduction . . . . . . . . . . . . . . . . . . . . . . . . . . . . . . 12

3.2 Problem Formulation . . . . . . . . . . . . . . . . . . . . . . . . 13

3.2.1 First-Order Markov Process . . . . . . . . . . . . . . . . . . . 15

3.2 .2 First-Order State Transition Matrix . . . . . . . . . . . . . . . 15

3.2 .3 Limitation of First-Order Process . . . . . . . . . . . . . . . . 17

3.2 .4 Second-Order Markov Process . . . . . . . . . . . . . . . . . . 17 
3.2 .5 Second-Order State Transition Matrix _. . . . . . . . . 18

3.3 Experimental Setup for Second-Order Markov Model . . . . . . . . . 20

3.3 .1 Error Metric . . . . . . . . . . . . . . . . . . . . 20

3.3.2 Transition Probability Computation and Prediction Results . 20

3.4 Limitation of Markov Model . . . . . . . . . . . . . . . . . . 25

4 Traffic Prediction Using Neural Network 26

4.1 Introduction . . . . . . . . . . . . . . . . . . . . . . . . 26

4.2 Recurrent Neural Network . . . . . . . . . . . . . . . . . . . . . . . . . . . . . . . $\quad 29$

4.3 Long-Short Term Neural Network . . . . . . . . . . . . . . . . 30

$4.4 \quad$ Ship Traffic Prediction with Neural Networks. . . . . . . . . . . . . . 32

4.5 Experimental Setup . . . . . . . . . . . . . . . . . . . . . . . . . . 34

$4.5 .1 \quad$ Error Metric . . . . . . . . . . . . . . . . . . . . . . . . . 35

4.5.2 Predicting Classes/Cell Number Corresponding to Future Location 36

4.5 .3 Predicting Continuous Value of Longitude and Latitude. . . . 38

4.5.4 Combining All These for A Bigger Region With More Data. . 46

4.6 Comparison with Markov Model . . . . . . . . . . . . . . . . . 48

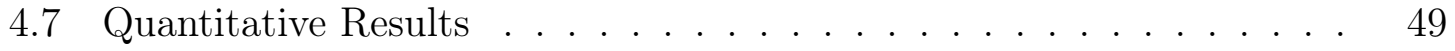

5 Conclusion \& Future Work 51

5.1 Markov Model . . . . . . . . . . . . . . . . . . . . . . . . . . . . . 51

5.2 Neural Network-Based Model . . . . . . . . . . . . . . . . . . . . 51

\begin{tabular}{ll}
\hline Bibliography & 53
\end{tabular} 


\section{List of Tables}

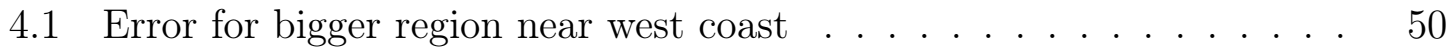

4.2 Error for bigger region near Gulf of Mexico . . . . . . . . . . . . . 50 


\section{List of Figures}

2.1 Raw data header, each row corresponds to a 1 AIS message. $\ldots$. . . . 7

$2.2 \quad$ ROI near the US coastal area limited by two points, highlighted by red

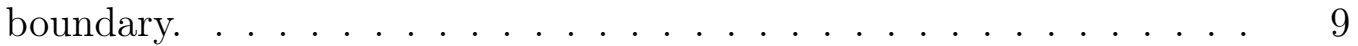

$3.1 \quad$ A simple example of Markov process with three states. . . . . . . . 13

3.2 Grid representation of ROI. . . . . . . . . . . . . . . . . . . . 14

3.3 Grid representation of ROI with names assigned to each cell. . . . . . 14

3.4 Intuition for first-order Markov process. . . . . . . . . . . . . 15

$3.5 \quad$ Next state of the vessel can be towards left or right if probability estimates for both are equal, which limits first-order Markov processes to incorporate direction of the vessel. . . . . . . . . . . . . . . 17

$3.6 \quad$ State diagram of second-order Markov model. . . . . . . . . . . . . 18

$3.7 \quad$ Visualization for computation of second-order transition matrix described in Section $3.3 .20 \ldots \ldots$. . . . . . . . . . . . . . . . . . 21

3.8 Visualization of a tuples described in Section 3.3 .2$] \ldots \ldots$. . . . . 21

3.9 Average error on testing dataset for Markov model of region 1. 53,234 trajectories were used to compute the transition probability. 14,201 trajectories (testing data) were used to compute the error. Top - 1 error after 4 hours is $11.7 \mathrm{~km}$. . . . . . . . . . . . . . . . . . . . . . . 24

3.10 Performance of Markov model on region 2. 52,249 trajectories were used to compute the transition probability. 13,063 trajectories (testing data) were used to compute the error. Top - 1 error after 4 hours is $17.7 \mathrm{~km}$. Because of diverging lanes, the average error is higher for this region. . . . . . . . . . . . . . . . . . . . 24 
3.11 Performance of Markov model on region 3. 46,558 trajectories were used to compute the transition probability. 11,640 trajectories (testing data) were used to compute the error. Top -1 error after 4 hours is

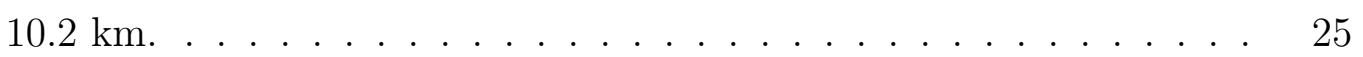

$4.1 \quad$ A simple example of Feed Forward Neural Network. . . . . . . . . . . 28

4.2 Basic unit of RNN. . . . . . . . . . . . . . . . . . . . . . . . . . . . . 29

4.3 Basic unit of LSTM. . . . . . . . . . . . . . . . . . . . . . . . . . . . 31

4.4 Architecture of proposed model. Number of units in each layer are selected according to [1]. . . . . . . . . . . . . . . . . . 34

4.5 Numbered representation of ROI with numbers assigned to each cell. 36

$4.6 \quad$ Error plot for cell prediction. The model was not able to learn the

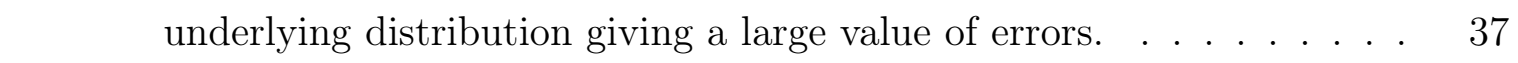

4.7 Error plot for Vanilla LSTM. Error after 4 hours is $7.9 \mathrm{~km}$. . . . . . 39

4.8 Comparison error plot on addition of type as a feature. Addition of \begin{tabular}{|c|}
\hline type as a time series input improves the performance of the model. \\
\hline
\end{tabular} Error after 4 hours is $6.6 \mathrm{~km}$. . . . . . . . . . . . . . . . . . . . 40

4.9 Comparison error plot on addition of length as a feature. Addition of length does not help to improve the performance. . . . . . . . . . . . 41

4.10 Destination assignment using clustering. . . . . . . . . . . . . . . . 41

4.11 Comparison error plot on addition of destination as a feature. Performance of model improves on addition of destination as a time series input. Average error after 4 hours drops to $5.4 \mathrm{~km}$. . . . . . . . . . . 42

4.12 Comparison error plot of two and three timestamps. Position coordinate of one more timestamp helps to improve the performace of the model. 43

4.13 Error plots for Region 2. The model's performance with type and destination as a feature is better compared to the Vanilla implementation. The model with position coordinates of three timestamps performs better than the position coordinates of two timestamps. . . . . . . . . 44

4.14 Error plots for Region 3. The model's performance with type and destination as a feature is better compared to the Vanilla implementation. Effect of destination is prominent because of diverging lanes. The model with position coordinates of three timestamps performs better than the position coordinates of two timestamps. . . . . . . . . . . . . . . 45 
4.15 Error on bigger region. Averge error for 30 minute prediction is $1.1 \mathrm{~km}$. Average error after 4 hours is $11.2 \mathrm{~km}$. . . . . . . . . . . . . . . . . 47

4.16 Error comparison on addition of regional SOG and COG. Addition of SOG and COG as a feature improves the peroformance of the model. The average after 4 hours drops to $8.2 \mathrm{~km}$. . . . . . . . . . . . . . . . 48

4.17 Error plot for bigger region near west coast. Orange color specifies more frequent routes, while light green color specifies less frequent routes. Average error after 4 hours is $12.18 \mathrm{~km}$. A total of 362,597 trajectories were used for the training, and 90,650 trajectories were used for the testing. . . . . . . . . . . . . . . . . . . . . . . . . . . . . . . . . . 48

4.18 Error plot for bigger region near Gulf. Orange color specifies more frequent routes, while light green color specifies less frequent routes. Average error after 4 hours is $10.91 \mathrm{~km}$. A total of 1, 436, 023 trajectories were used for the training, and 359,006 trajectories were used for the testing. Due to large number of training trajectories average error is less than the west coast region. . . . . . . . . . . . . . . . . . . . . . 49

4.19 Error comparison of Markov model and LSTM based model. The Top - 1 error for the Markov model increases faster compared to LSTM based model for the long term prediction. . . . . . . . . . . . . . . . 49 


\section{Chapter 1}

\section{Introduction}

\subsection{Motivation}

The ability to predict future locations of ocean vessels is of paramount importance to maritime situational awareness and maritime situational planning. Ambient noise forecasting is a critical element of sonar performance prediction. As maritime vessels and ships also constitute ocean noise, ship traffic plays an important role in estimating noise level estimates along with the weather and other biological activities. We focus on the prediction of ship traffic as a motivating problem. Our goal is to predict the future location of maritime vessels, which is useful to predict the traffic in the ocean. The Automatic Identification System (AIS) is an untapped resource that provides real-time ship locations. In this thesis, we use historical AIS data to model the vessel's behavior in a given Region of Interest (ROI), which can be used with real-time AIS data stream to predict the vessel's future location.

Methods to predict future locations of the vessel can be classified into two types. The first type tries to model the vessel's physical behavior and works on an assumption that the next location of the vessel would be dependent upon its current location, i.e., it uses current location and speed of the vessel to predict the next locations [2]. The goal of these methods is to develop a mathematical formula that takes current location, speed, and heading information to predict the vessel's future locations. The

problem with these methods is that due to a lot of variability in the open seas, these 
methods are not good for long-term prediction, i.e., prediction for more than 10 minutes [2]. The second type of methods are data driven methods [3, 4, 1]. Since the compulsion of the AIS-based system, every modern vessel is equipped with one. An AIS-based system, which transmits the vessel's location at a regular interval of time. The advantage of these systems is the accumulation of massive amounts of data, which is available to make some sense of the position coordinates of the vessels. The second strategy uses these historical AIS data to develop a method used to make predictions. The methods described in this thesis also take advantage of this historical AIS data and current data to make predictions in future timestamps.

\subsection{Current State of The Art}

Methods to predict the next state from the current state of the system assume that the next state of the vessel is dependent upon the current state of the system. The method presented in [2] uses an extended Kalman filter-based approach that uses adaptive filtering algorithms to predict the next location of the vessel, which estimates values of velocity and acceleration, which are used to make prediction for the next location. Though this method is effective for short term prediction, i.e., prediction of 5 or 10 minutes, it fails for long term prediction, i.e., prediction for more than 30 minutes.

Data driven approaches are of two types. The first type consists of methods, where traffic route patterns are extracted for a given ROI. The vessel's future location is computed by traversing on one of the traffic routes on which the vessel is traveling, using the vessel's current velocity and direction. Methods to extract traffic route patterns are given in [5] [6] [7]. The method called Traffic Route Extraction and Anomaly Detection (TREAD) is proposed in [5], which uses a density-based clustering

algorithm called DBSCAN [8] to extract the traffic route pattern. The method 
described in [6] uses Ornstein-Uhlenbeck processes and DBSCAN-based [8] algorithms to generate graph representation of maritime traffic. The method proposed in [3] uses traffic routes for a given ROI extracted by the methods shown in [5]. The current trajectory is given to a clustering algorithm called K-Nearest Neighbors (KNN) 9] to classify the trajectory to one of the extracted traffic routes. Constant velocitybased model or particle filter-based model is used to predict the future location of the vessel along the selected traffic route. The method proposed in [4] models the vessel's motion dynamics using Ornstein-Uhlenbeck processes. Ornstein-Uhlenbeck processes is a stochastic process, which is used to model the Brownian motion [10]. Proposed method in [4] uses route information extracted by [5] and historical AIS data to come up with the optimal parameter of Ornstein-Uhlenbeck processes. Each traffic route extracted corresponds to typical motion behaviour, which serves as an input to Ornstein-Uhlenbeck tracking model to predict the vessel's future location. The method described in [4] performs good on straight traffic routes but fails on complex traffic areas. Constant velocity-based method described in [3] does not perform well when predicting for more than 1 hour, while the particle filter-based model is computationally expensive and difficult to implement.

In [11], authors have given an algorithm called Single Point Neighbour Search Method, which uses all the points in the AIS dataset, which are in close proximity to the current location of the vessel. Number of these points, which are near to current location, are bounded by distance parameter $r$ (haversine distance) [12] and maximum deviation of course and speed. Priori course and speed for the future location is taken as the mean of course and speed of these close neighboring points. This priori course and speed are used to compute posterior course and speed. These posterior course and speed along with step length $\Delta l$ are used to propagate the vessel to compute the future location of the vessel. The method proposed in [13] first extracts waterway 
patterns using a lattice-based DBSCAN algorithm. After the extraction of waterway patterns, Kernel Density Estimation (KDE) [14 based model is made for a particular trajectory, which keeps track of the amount of distance traveled within intervals of the last half an hour. Based on that, KDE distribution is computed, whose mean value is used to traverse the distance along the waterway pattern to compute the future location of the vessel. Both methods [11] [14] are limited by their ability to predict for more than 60 minutes.

The second type of data driven approaches are based on machine learning and neural networks. The method proposed in [15] first finds factors that correlate with future locations and uses modelling technique called Support Vector Machines (SVM) [16] to predict the future location of the vessels. Exploiting the time-series nature of the data [1] uses position coordinates of the previous 90 minutes at an interval of 1 minute as a feature for LSTM-based network to predict the future location. The method proposed in [17] trains an embedding layer, which is made up of VRNN [18], and takes the discretized value of the current position coordinates, Speed Over Ground (SOG), and Course Over Ground (COG) as features to learn the embedding layer, which is later used to predict the next locations of the vessel. The method proposed in [19] takes the trajectory data of one trajectory and trains the LSTM-based network for a single trajectory to make a prediction. The method described in [1], due to its problem setup can't be used to do back-to-back prediction. The method described in [17] fails to talk about use of the embedding layer for the task of prediction. The method proposed in [19] is limited for a single trajectory and needs a separate neural network for each trajectory. 


\subsection{Contributions}

\subsubsection{Markov Model}

The Markov-based model described in Chapter 3 uses historical AIS data to compute the conditional probability of next location given current location or current and previous location for a given ROI. We provide a simple approach to compute transition probabilities for a given ROI, which can be used with the vessel's initial location vector to compute the vessel's future location by simple matrix multiplication involving a sparse matrix. We have defined an error metric to assess the prediction model's performance for various regions near the Los Angeles coastal area.

\subsubsection{Neural Network-Based Model}

We propose an LSTM-based neural network model, which is similar to [1], which incorporates the position of the vessel and other side information such as type, destination and regional Speed Over Ground (SOG) and Course Over Ground (COG) to predict future locations of the vessel. We demonstrate the effects of various features on the performance of the model. We define the error metric to assess the model's ability to predict the vessel's future location multiple timestamps in the future. Unlike [1, our proposed model can be used to do back-to-back predictions. Apart from that, the proposed model also takes into account features like destination and month of trajectory, which helps to improve the performance of the model. 


\section{Chapter 2}

\section{Data Pre-processing}

\section{$2.1 \quad$ AIS Dataset}

The dataset used in this work is available publicly at [20]. The dataset has AIS messages for the entire US coastal area from year 2009 to 2018. The files are available on a yearly basis. For each individual year, the files are grouped according to zone and month. The dataset has files for 18 different zones, giving a total of $18 \times 12=216$ files for an individual year. Every file out of these 216 files has the format of AIS_YYYY_MM_ZoneNN.zip for better organization, where YYYY corresponds to the year, MM corresponds to month, and NN corresponds to zone number. Individual files are made up of AIS messages from vessels of all types filtered based on year, month, and zone.

\subsection{AIS Message}

An Automatic Identification System (AIS) is a system that sends out messages related to ship voyage on every few time stamps. The time difference between two messages can be between a few seconds to tens of minutes to hours. These messages carry a wealth of information related to the vessels'/ships' status, which maritime authorities and neighbouring vessels/ships use to avoid traffic congestion and collision. AIS messages from multiple sources are collected and transformed into human-readable form to make an individual file of AIS dataset. The data is stored in files with a 


\begin{tabular}{|c|c|c|c|c|c|c|c|c|c|c|c|c|c|c|c|}
\hline & MMSI & BaseDateTime & LAT & LON & SOG & COG & Heading & VesselName & IMO & CallSign & VesselType & Status & Length & Width & Draft \\
\hline 0 & 367114690 & $\begin{array}{r}\text { 2017-01- } \\
01 \text { T00:00:06 }\end{array}$ & 48.51094 & -122.60705 & 0.0 & -49.6 & 511.0 & $\mathrm{NaN}$ & $\mathrm{NaN}$ & $\mathrm{NaN}$ & $\mathrm{NaN}$ & $\begin{array}{r}\text { under } \\
\text { way } \\
\text { using } \\
\text { engine }\end{array}$ & $\mathrm{NaN}$ & $\mathrm{NaN}$ & $\mathrm{NaN}$ \\
\hline 1 & 367479990 & $\begin{array}{r}2017-01- \\
01 T 00: 00: 03\end{array}$ & 48.15891 & -122.67268 & 0.1 & 10.1 & 353.0 & $\begin{array}{r}\text { WSF } \\
\text { KENNEWICK }\end{array}$ & IMO9618331 & WDF6991 & 1012.0 & moored & 83.39 & 19.5 & 3.2 \\
\hline
\end{tabular}

Figure 2.1: Raw data header, each row corresponds to a 1 AIS message.

.csv extension, which makes data available in tabular format, i.e., on extracting the AIS_YYYY_MM_ZoneNN.zip file, we get AIS_YYYY_MM_ZoneNN.csv file. An example of the raw data is shown in Fig. 2.1.

The Pandas package of python [21] has been used in this work to read and preprocess the data. Each row of the CSV file corresponds to one AIS message. One message carries information of the one vessel at one particular time. The data have the following 16 columns:

1. MMSI - Corresponds to the identity of the vessel, unique for a vessel

2. BaseDateTime - Timestamp of the message

3. LAT - Latitude coordinate of the vessel at the current timestamp

4. LON - Longitude coordinate of the vessel at the current timestamp

5. SOG - Speed Over Ground of the vessel at the current timestamp

6. COG - Course Over Ground of the vessel at the current timestamp

7. Heading - Heading of the vessel at the current timestamp

8. VessleName - Corresponds to the identity of the vessel

9. IMO - Corresponds to the identity of the vessel

10. CallSign - Corresponds to the identity of the vessel

11. VesselType - Type of the vessel 
12. Status - Current Status of the vessel

13. Length - Length of the vessel

14. Width - Width of the vessel

15. Draft - Size of Draft of the vessel

16. Cargo - Capacity of the vessel if its of type Cargo

Each AIS message contains two types of information.

1. Static - Does not change over the vessel's trajectory

2. Dynamic - Changes over the vessel's trajectory

MMSI, VessleName, IMO, CallSign, Length, Width, Draft, Cargo, and VesselType are static type of data, which remains same for a particular trajectory. Where as LAT, LON, SOG, COG, Heading, and Status are dynamic types of data, which change over time for a particular trajectory.

\subsection{Data Pre-Processing}

For the task of vessel trajectory prediction, raw data needs to be processed before applying any modelling techniques. This section deals with steps of pre-processing the data so that modelling can be done in an efficient and organized way. 


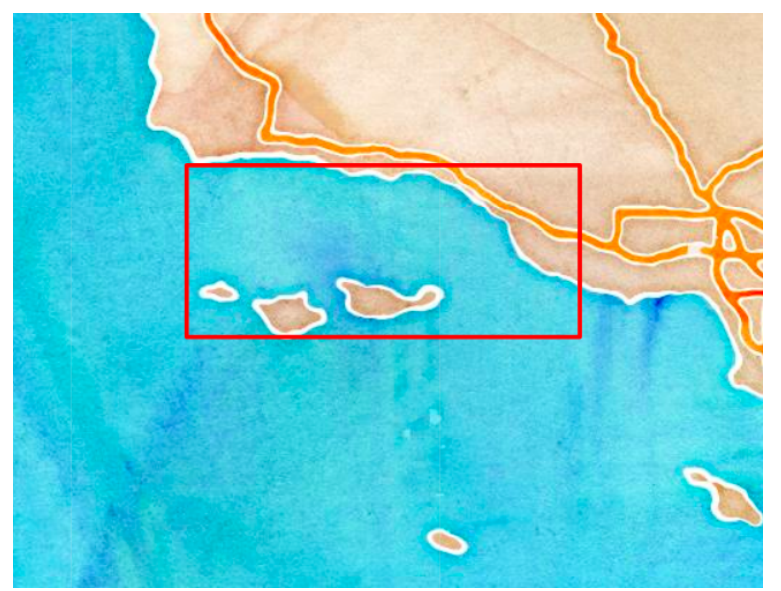

Figure 2.2: ROI near the US coastal area limited by two points, highlighted by red boundary.

1. Segregate data for desired region.

The first step of pre-processing is to segregate the data, which is limited to the Region of Interest (ROI). Each ROI is assumed to be a rectangular region and is characterized by two points. Two geographic coordinates, i.e., bottom left point and top right point of the rectangular region, are used to characterize the region. Doing so helps reduce the memory burden on the system and make the problem more tractable. This step crops the data which is limited to ROI as shown by RED borders in Fig. 2.2.

2. Dropping unnecessary columns.

Static information such as VesselName, IMO, CallSign can not be attributed to vessel's general behaviour. Apart from that, there are large numbers of missing entries in Width, Draft, Cargo, and Status column of the data. So this step drops all these unnecessary columns to reduce memory usage for the next pre-processing steps.

3. Sorting the reduced data with respect to time.

As timestamps of the AIS message in a file may not be sorted in increasing order 
of time. This step sorts the messages with respect to time.

4. Generate a list of vessels.

MMSI is used to identify individual vessels as it is unique in nature. This step generates a list of all the vessels, i.e., MMSI entries, which has at least one message in the reduced data.

5. Segregate data of an individual vessel.

After generating a list of the vessel, this step segregates data for the individual vessel. Corresponding to every vessel, one CSV file gets generated, which has data only for one specific vessel.

6. Generate vessel type file.

This step assigns type information to every vessel in the list generated in step 4. This work focuses on two types of vessels: Tanker and Cargo. This step generates one list for Tanker vessels, and one list for Cargo vessels.

7. Generating vessel trajectory files.

This step generates separate trajectories from the files generated in step 5 . Corresponding to one MMSI, there may be more than one instance of travel in given ROI over the period of years. This step breaks the file corresponding to one MMSI into multiple trajectory files. The decision to break the trajectory is dependent upon the time difference between consecutive AIS messages. If the time difference of consecutive AIS messages is more than 30 minutes, then those messages become part of two different trajectories.

8. Augment trajectory data. 
This step augments the trajectory data of one continuous trajectory into multiple trajectories sampled at an interval of 30 minutes. For example, consider a trajectory of 2 hours, From $12: 00: 00$ to $14: 00: 00$, with AIS messages at an interval of 1-2 minutes. This step generates 15 trajectories from this one trajectory. The first trajectory would have messages with timestamp $12: 00: 00$, $12: 30: 00,13: 00: 00,13: 30: 00,14: 00: 00$. The second trajectory would have messages with a timestamp, which differs by 2 minutes, i.e., 12 : 02 : 00, $12: 32: 00,13: 02: 00,13: 32: 00$. Likewise there would be a total 15 trajectories. The last trajectory would have messages with a timestamp, which differs by 28 minutes, i.e., $12: 28: 00,12: 58: 00,13: 28: 00,13: 58: 00$.

9. Compute median $S O G$ and $C O G$ for rectangular grid.

For a rectangular region, this step computes the median value of SOG and COG for all cells in that region. Cell is defined as one small region of a grid-like structure, which gets generated by dividing ROI with horizontal and vertical lines. This step computes the matrix of the median SOG and median COG for specific types of vessel.

After pre-processing, we get a set of trajectories for a given ROI. Along with trajectories, we also get two matrices, which store median values of SOG and COG, respectively. These pre-processed data can be directly used to compute the transition probability matrix, specified in Chapter 3. Similarly, this data can directly be used to make a dataset in the form $x$ (input features) and $y$ (output), which can be used to train the neural network-based model specified in Chapter 4. 


\section{Chapter 3}

\section{Markov Model}

\subsection{Introduction}

The Markov chain model is a useful tool to model sequential data. The key idea behind the Markov model is that the next data and current data in the sequence can be considered as one of the states of the Markov model. To model the ship location prediction problem using a Markov chain, we consider each location a state. Predicting the next data in a sequence is equivalent to determining probabilistic next states from the system's current state. A Markov process is a random process that satisfies Markov property, which is defined as follows. Consider a system with states $x_{0}, x_{1}$, $x_{2}, x_{3}, \ldots, x_{n-1}$, i.e., a total of $n$ states. The system can go from state $x_{i}$ to $x_{j}$, where $i, j=0,1, \ldots, n-1$. The Markov property states that the next state of the system depends only upon the current state and not on the previous states. Formally,

$$
P\left(x_{j} \mid x_{i}, x_{i-1}, x_{i-2}, \ldots\right)=P\left(x_{j} \mid x_{i}\right) .
$$

The dynamics of the system can be characterized by a matrix called state transition matrix $P \in \mathbb{R}^{n \times n}$. where the element $P_{i j}$ equals the probability of transitioning from state $i$ to state $j$. Values of state transition probabilities can be estimated from historical data. In this work, we have used historical AIS data to compute the values of the transition probability matrix. With the initial state and state transition matrix, 


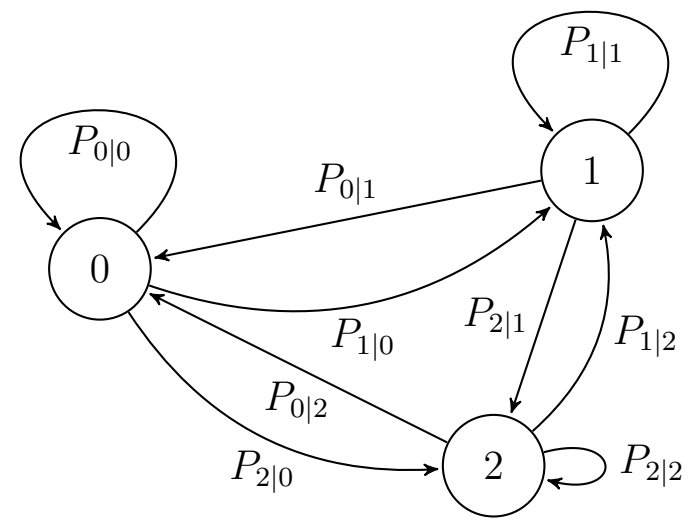

Figure 3.1: A simple example of Markov process with three states.

we can obtain a probabilistic estimate of the next state of the system.

Fig. 3.1 is an example of a Markov process with three states 0,1 , and 2 . The process can be described by a state transition matrix

$$
P=\left[\begin{array}{ccc}
P_{0 \mid 0} & P_{1 \mid 0} & P_{2 \mid 0} \\
P_{0 \mid 1} & P_{1 \mid 1} & P_{2 \mid 1} \\
P_{0 \mid 2} & P_{1 \mid 2} & P_{2 \mid 2}
\end{array}\right],
$$

where the element $P_{i j}=P_{j \mid i}$ represents the probability of going from state $i$ to state $j$.

\subsection{Problem Formulation}

The problem of vessel trajectory prediction can be formulated as a Markov process as follows. Consider the given Region of Interest (ROI). The region of interest can be assumed to be a rectangular region and is characterized by two points. Divide the ROI with lines of constant latitude horizontally (parallel to the equator) and divide ROI with lines of constant longitude (perpendicular to the equator) to get a grid like representation of the ROI as in Fig. 3.2. With such a grid, vessel locations can be parameterized according to which cell they lie in. We assign a state to each cell of a grid in a sequential fashion. Starting from the first row at the top, we assign states in 


\section{LonMax, LatMax (Top Right)}

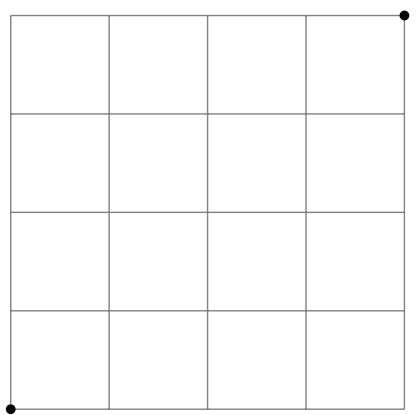

LonMin, LatMin (Bottom Left)

Figure 3.2: Grid representation of ROI.

LonMax, LatMax (Top Right)

\begin{tabular}{|l|l|l|l|}
\hline$x_{0}$ & $x_{1}$ & $x_{2}$ & $x_{3}$ \\
\hline$x_{4}$ & $x_{5}$ & $x_{6}$ & $x_{7}$ \\
\hline$x_{8}$ & $x_{9}$ & $x_{10}$ & $x_{11}$ \\
\hline$x_{12}$ & $x_{13}$ & $x_{14}$ & $x_{15}$ \\
\hline
\end{tabular}

LonMin, LatMin (Bottom Left)

Figure 3.3: Grid representation of ROI with names assigned to each cell.

increasing order while going from left to right. After assigning the states to the right most cell, we go to the next row at the bottom and continue assigning states from left to right. After reaching to the bottom right cell, we get states assigned to every cell of the grid as in Fig. 3.3 .

Based on the vessel's position in any of the cells, it can be considered that the vessel is in the state corresponding to that cell. Here, the state space for this Markov process consists of states $x_{0}, x_{1}, x_{2}, \ldots, x_{n-1}$. Suppose coordinates of the vessel correspond to cell $x_{5}$ in Fig. 3.3, then we say that the Markov process corresponding to vessel trajectory is in state $x_{5}$. Now the prediction problem is equivalent to finding the next 


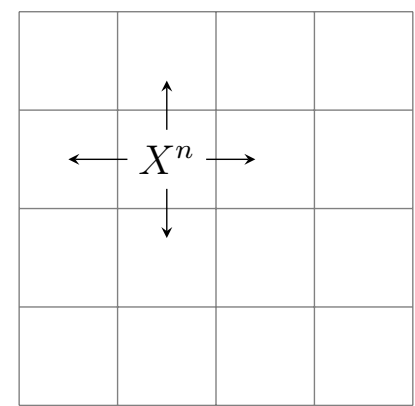

Figure 3.4: Intuition for first-order Markov process.

state of this Markov process.

\subsubsection{First-Order Markov Process}

While computing the next state, if we consider only the current state, then the Markov process is called a first-order Markov process, which can be described by the following equation.

$$
P\left(X^{n+1} \mid X^{n}, X^{n-1}, X^{n-2}, \ldots ., X^{0}\right)=P\left(X^{n+1} \mid X^{n}\right),
$$

where $X^{n}$ denotes the state of the vessel at time $n$.

\subsubsection{First-Order State Transition Matrix}

Consider Fig. 3.4, current state $X^{n}$ of vessel corresponds to the vessel's current position coordinates. Now the vessel can go from its current state $X^{n}$ to any of the possible states $x_{0}, x_{1}, x_{2}, x_{3}, \ldots, x_{n-1}$ which can be called next state of the vessel, i.e., $X^{n+1}$. From historical AIS data of the vessels, we can compute the probability of the vessel going from one cell to another. If we put all those probabilities in matrix form, what we get is called a first-order state transition matrix. 


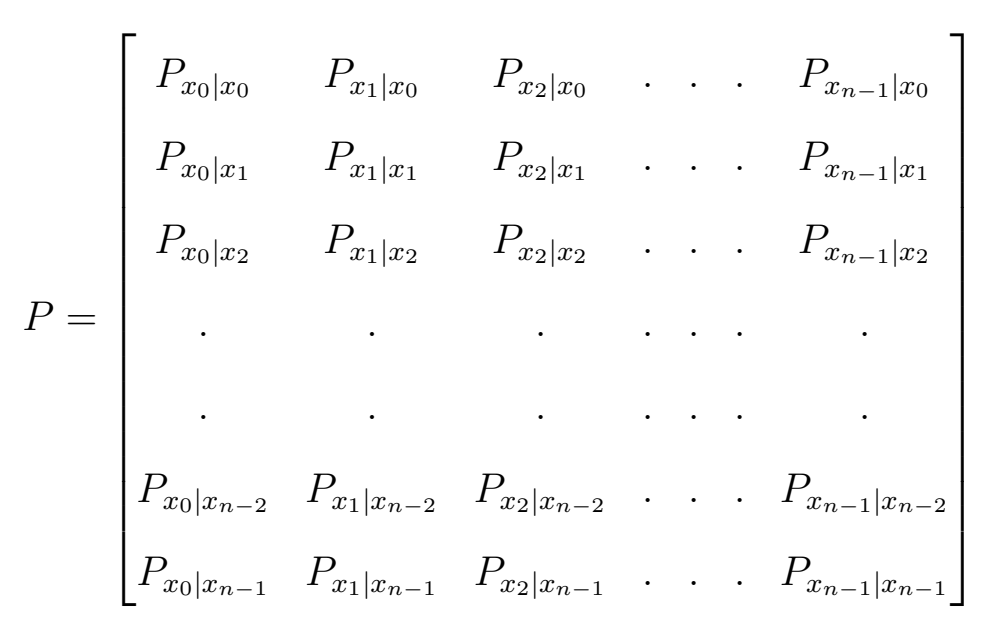

A first-order state transition matrix is denoted by $P \in \mathbb{R}^{n \times n}$, where individual element $P_{i j}$ represents the probability of going from state $i$ to state $j$. The State vector corresponding to position of the vessel at time $t$ is represented by $\pi^{t}$. State vector is a vector in $\mathbb{R}^{n}$, which has a probability value corresponding to each state of the system. $\pi^{0}$ is known as the initial state of the system. and is encoded as a one hot vector corresponding to the (known) current location of the vessel. For example, the vessel in cell $x_{5}$ of Fig. $3.3, \pi^{0}$ can be given by $e_{5}$. where $e_{i}$ denotes the $i^{\text {th }}$ standard basis vector.

The probabilistic position after one timestamp can be computed using a state transition matrix and initial state vector as follows

$$
\pi^{1}=P^{T} \pi^{0}
$$

This can be generalized to $n$ time stamps as follows

$$
\pi^{n}=P^{T} \pi^{n-1}
$$

The state with the maximum value of probability in the state vector $\pi^{(1)}$ after one timestamp is considered as predicted state after one timestamp. The center point 


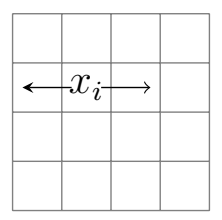

Figure 3.5: Next state of the vessel can be towards left or right if probability estimates for both are equal, which limits first-order Markov processes to incorporate direction of the vessel.

corresponding to this state's cell is considered the predicted location after timestamp. Suppose after one timestamp state $x_{3}$ comes out to be most probable, then the center point of cell $x_{3}$ becomes the predicted location after one timestamp.

\subsubsection{Limitation of First-Order Process}

The problem with the first-order Markov model is that it cannot keep track of direction, which can be seen in Fig. 3.5. Another disadvantage is that the first-order Markov model cannot incorporate a sense of velocity in the transition probability matrix. To overcome these limitations, memory needs to be added to the system. To include memory into the system, higher order Markov models can be used.

\subsubsection{Second-Order Markov Process}

A second-order Markov process differs from first order in a way that it helps to incorporate memory into the system. For a second-order process, the next state of the process is not only dependent upon the current state, but it also depends upon the previous state from which the system has come to the current state. Mathematically, this can be represented as follows

$$
P\left(X^{n+1} \mid X^{n}, X^{n-1}, X^{n-2}, \ldots . ., X^{0}\right)=P\left(X^{n+1} \mid X^{n}, X^{n-1}\right) .
$$

The dynamics of the system can be characterized by the current state, previous state, and the matrix called second-order state transition matrix. 

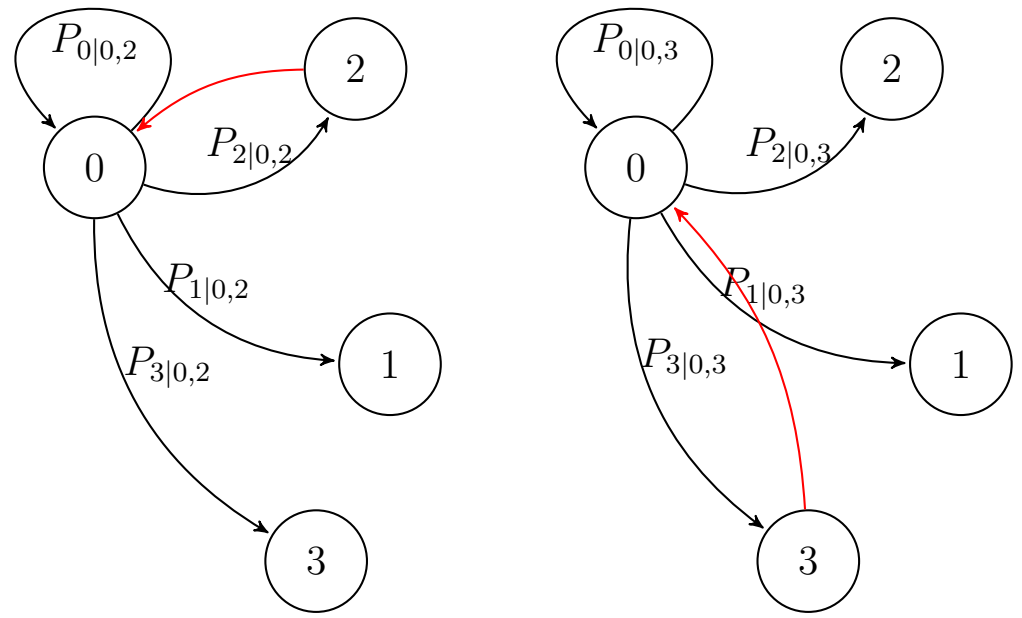

Figure 3.6: State diagram of second-order Markov model.

\subsubsection{Second-Order State Transition Matrix}

The state diagram of the second-order Markov model with four states can be represented by Fig. 3.6. The transition from the previous state to the current state is indicated by a red arrow. The next state of the system is dependent upon current state 0 , as well as the state from which it has come to the current state, i.e., 2 and 3.

The dynamics of the system can be characterized by a second-order state transition matrix $P \in \mathbb{R}^{n^{2} \times n}$, For Fig. 3.6, state transition matrix can be given as 


$$
P=\left[\begin{array}{llll}
P_{0 \mid 0,0} & P_{1 \mid 0,0} & P_{2 \mid 0,0} & P_{3 \mid 0,0} \\
P_{0 \mid 1,0} & P_{1 \mid 1,0} & P_{2 \mid 1,0} & P_{3 \mid 1,0} \\
P_{0 \mid 2,0} & P_{1 \mid 2,0} & P_{2 \mid 1,0} & P_{3 \mid 2,0} \\
P_{0 \mid 3,0} & P_{1 \mid 3,0} & P_{2 \mid 3,0} & P_{3 \mid 3,0} \\
P_{0 \mid 0,1} & P_{1 \mid 0,1} & P_{2 \mid 0,1} & P_{3 \mid 0,1} \\
P_{0 \mid 1,1} & P_{1 \mid 1,1} & P_{2 \mid 1,1} & P_{3 \mid 1,1} \\
P_{0 \mid 2,1} & P_{1 \mid 2,1} & P_{2 \mid 2,1} & P_{3 \mid 2,1} \\
P_{0 \mid 3,1} & P_{1 \mid 3,1} & P_{2 \mid 3,1} & P_{3 \mid 3,1} \\
P_{0 \mid 0,2} & P_{1 \mid 0,2} & P_{2 \mid 0,2} & P_{3 \mid 0,2} \\
P_{0 \mid 1,2} & P_{1 \mid 1,2} & P_{2 \mid 1,2} & P_{3 \mid 1,2} \\
P_{0 \mid 2,2} & P_{1 \mid 2,2} & P_{2 \mid 2,2} & P_{3 \mid 2,2} \\
P_{0 \mid 3,2} & P_{1 \mid 3,2} & P_{2 \mid 3,2} & P_{3 \mid 3,2} \\
P_{0 \mid 0,3} & P_{1 \mid 0,3} & P_{2 \mid 0,3} & P_{3 \mid 0,3} \\
P_{0 \mid 1,3} & P_{1 \mid 1,3} & P_{2 \mid 1,3} & P_{3 \mid 1,3} \\
P_{0 \mid 2,3} & P_{1 \mid 2,3} & P_{2 \mid 2,3} & P_{3 \mid 2,3} \\
P_{0 \mid 3,3} & P_{1 \mid 3,3} & P_{2 \mid 3,3} & P_{3 \mid 3,3}
\end{array}\right],
$$

where $P_{k \mid j, i}$ is defined as probability of going to state $k$, if current state is $j$ and previous state is $i$. For a system with states $x_{0}, x_{1}, x_{2}, x_{3}, \ldots, x_{n-1}$, the transition probability matrix $P$ can be generalized. Using matrix $P$ and initial position vector, we can compute the future location of the vessels. Probabilistic future position, i.e., next state of the Markov model $\pi^{t+1} \in \mathbb{R}^{n}$, can be computed from the current position i.e. $\pi^{(t)}$ and previous position $\pi^{(t-1)}$.

$$
\pi^{t+1}=P^{T} \pi^{(t,(t-1))}
$$

where $\pi^{(t,(t-1))} \in \mathbb{R}^{n^{2}}$ is generated from $\pi^{t}$ and $\pi^{t-1}$, which is an ordered pair of the 
two position vectors. $\pi^{(0)}$ and $\pi^{(1)}$ correspond to the initial two locations of the vessel.

\subsection{Experimental Setup for Second-Order Markov Model}

After pre-processing the data with the method described in Section 2.3 for a given ROI, we used the trajectory data to compute the second-order transition probability matrix $P$. The computation of the transition probability matrix happens offline. After the transition probability matrix is computed, we used it directly to make a prediction in a given ROI. We computed the transition probability matrix for several regions of similar sizes near the US coastal area to verify the generability of the method.

\subsubsection{Error Metric}

As this method gives the prediction in terms of probability, we have defined an error metric to assess the performance of the model. Top $-K$ error for a particular trajectory is defined as the minimum value of haversine distance [12] between actual position coordinates of the vessel and top $K$ predictions. Corresponding to the next state vector $\pi^{t+1}$, states with maximum probability value are sorted in descending order. The Center point of these states are used as predicted locations. Based on the value of $K$, first $K$ distances are computed between the actual location and predicted locations. The minimum value of these distances is used as an error for one particular trajectory. Doing these on every trajectory in the testing dataset and taking mean gives us Top $-K$ error for that particular timestamp. Note that the Top -1 error corresponds to the case, where the predicted location is always the most probable.

\subsubsection{Transition Probability Computation and Prediction Results}

Computation of transition probability can be visualized by Fig. 3.7 3.8, The preprocessed data consisted of trajectories, which carries the vessel's location at an 

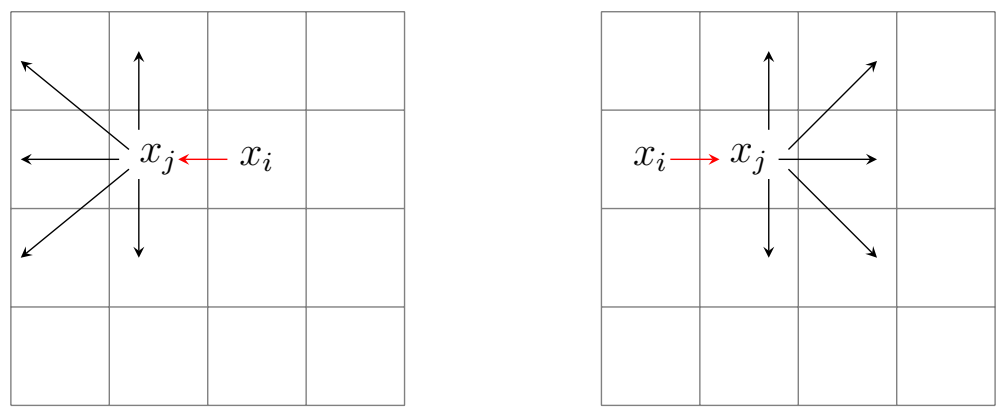

Figure 3.7: Visualization for computation of second-order transition matrix described in Section 3.3 .2 .
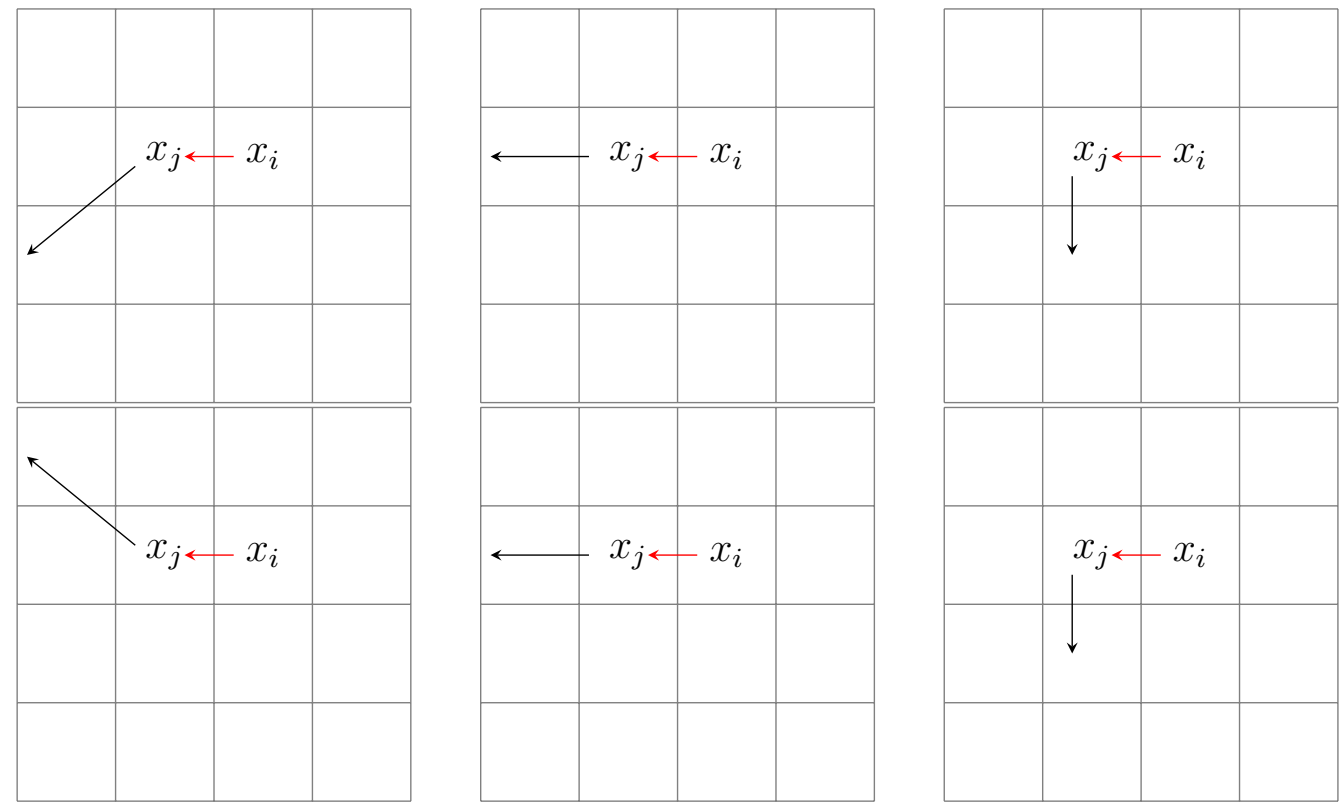

Figure 3.8: Visualization of a tuples described in Section 3.3.2.

interval of 30 minutes. We computed the transition probability matrix for a region characterized by

1. $L O N_{-} M I N=-120.50$,

2. $L O N_{-} M A X=-119.00$,

3. $L A T \_M I N=33.90$,

4. $L A T T_{-} M A X=34.44$. 
After dividing the region with a grid size of $0.1 * 0.1$ degrees, the total states for this ROI came out to be 8100 . Now every trajectory is considered a sequence of states. Denote sequence of these trajectory states by $x_{0}, x_{1}, \ldots, x_{n-1}$. Now break the continuous sequence in tuples of three states

$$
\begin{gathered}
{\left[x_{0}, x_{1}, x_{2}\right]} \\
{\left[x_{1}, x_{2}, x_{3}\right]} \\
{[., ., .]} \\
{\left[x_{n-3}, x_{n-2}, x_{n-1}\right]}
\end{gathered}
$$

One instance of which in a general form, can be written as $\left[x_{i}, x_{j}, x_{k}\right]$, which represents a vessel going from $x_{i}$ to $x_{j}$ to $x_{k}$. Now $x_{i}$ can take values from 0 to 8099 , similarly $x_{j}$ and $x_{k}$ can take values from 0 to 8099 .

A row of transition probability matrix corresponds to every possible combination of $\left(x_{i}, x_{j}\right)$ ordered pairs encountered in the training data. We start with the empty list, for every combination of $\left(x_{i}, x_{j}\right)$ encountered, we append the dictionary in the list. For the set of tuples shown in Fig. 3.8, there will be one dictionary in the list. Now for one such combination of $\left(x_{i}, x_{j}\right)$, keys in the dictionary corresponds to state values of possible values of $x_{k}$. For Fig. 3.8, dictionary corresponding to $\left(x_{i}, x_{j}\right)$ would be identified by an ordered pair of $(6,5)$. Now on the first encounter of a specific value of $x_{k}$, that specific value of $x_{k}$ is appended to keys of the dictionary with value 1 . On every subsequent encounter of $x_{k}$, the value is incremented by 1 . For an ordered pair of $(6,5)$, starting from top left of Fig. 3.8. first $x_{k}=8$ is encountered, giving $\operatorname{Dict}_{(6,5)}=\{8: 1\}$. After processing the next tuple $\left(x_{k}=4\right)$, we get $\operatorname{Dict}_{(6,5)}=\{8: 1,4: 1\}$. After processing the next tuple, we get $\operatorname{Dict}_{(6,5)}=\{8: 1,4: 1,9: 1\}$. Similarly after processing the all 
six tuples, we get $\operatorname{Dict}_{(6,5)}=\{8: 1,4: 2,9: 2,0: 1\}$. Repeating this process for every trajectory gives us a list of dictionaries, which represents pairs of the previous state and the current state. Now all the keys for a dictionary representing $\left(x_{i}, x_{j}\right)$ gives the information about possible next states. By adding values corresponding to every key of a dictionary representing $\left(x_{i}, x_{j}\right)$, gives us all transitions for the combination of $\left(x_{i}, x_{j}\right)$. Lets call this sum as TotalTransition. Dividing every value corresponding to every key of a dictionary representing $\left(x_{i}, x_{j}\right)$ with TotalTransition, gives us values of transition probabilities. This list is converted into a sparse representation of transition probability matrix. Once computed, this matrix is used to make predictions.

The previous location of each vessel is transformed into a one hot-encoded vector, i.e., $\pi^{(t-T)} \in \mathbb{R}^{n}$ is a state vector corresponding to the previous location ( $T$ minutes back); similarly, the current location of the vessel is transformed into one hot encoded vector, i.e., $\pi^{(t)} \in \mathbb{R}^{n}$ is a state vector corresponding to the current location. Combining $\pi^{(t-T)}$ and $\pi^{(t)}$ and multiplying it with transition probability matrix gives probabilistic state vector, which can be used to compute future location

$$
\pi^{(t+T)}=P^{T}\left(\pi^{(t,(t-T))}\right)
$$

where the value of $T$ is 30 minutes. $\pi^{(t,(t-T))} \in \mathbb{R}^{n^{2}}$ is an ordered pair of $\pi^{(t-T)}$ and $\pi^{(t)}$. Similarly $\pi^{(t+T)}$ and $\pi^{(t)}$ can be used to compute $\pi^{(t+2 T)}$, which can be generalized to multiple timestamps in the future.

For a given region, we computed the transition probability matrix with the above specified method, and computed Top $-K$ error on the testing dataset. The region had 53, 234 trajectories for computation of transition probability, and 14, 201 trajectories were used for the testing purpose. Top $-K$ error can be seen in Fig. 3.9, Top -1 error after 4 hour is $11.7 \mathrm{~km}$. On increasing the value of $K$, error reduces significantly; 


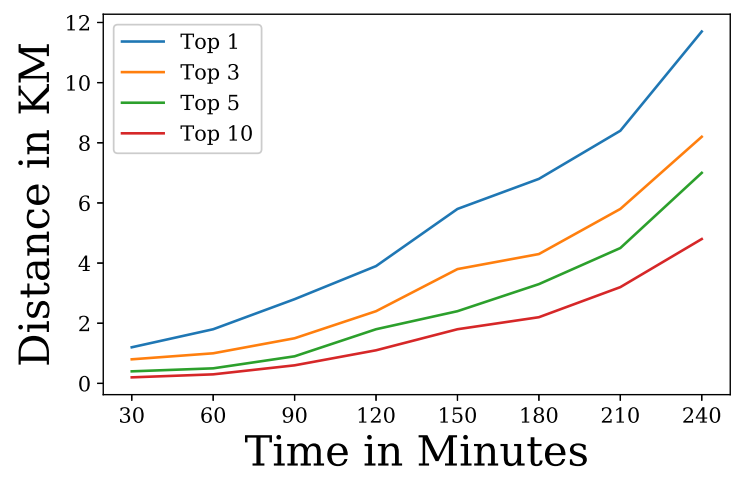

Figure 3.9: Average error on testing dataset for Markov model of region 1. 53, 234 trajectories were used to compute the transition probability. 14, 201 trajectories (testing data) were used to compute the error. Top -1 error after 4 hours is $11.7 \mathrm{~km}$.

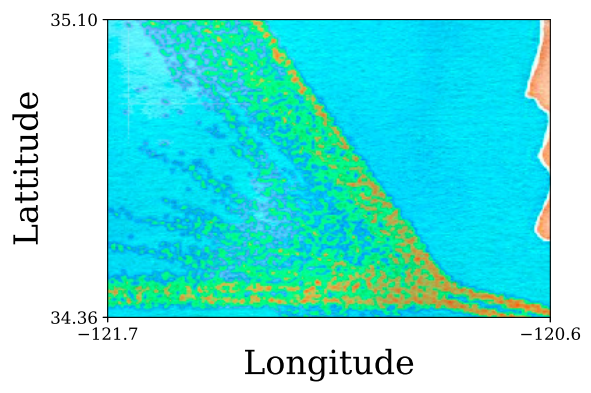

(a) Heatmap of region 2. Orange color specifies more frequent routes, while light green color specifies less frequent routes.

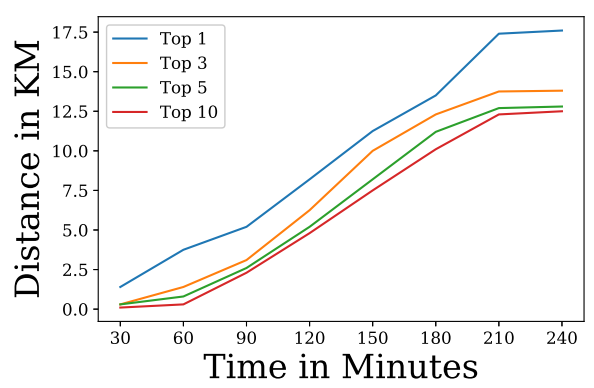

(b) Average error on testing dataset

Figure 3.10: Performance of Markov model on region 2. 52, 249 trajectories were used to compute the transition probability. 13, 063 trajectories (testing data) were used to compute the error. Top - 1 error after 4 hours is $17.7 \mathrm{~km}$. Because of diverging lanes, the average error is higher for this region.

for the same region, Top -3 and Top -5 errors are $8.2 \mathrm{~km}$ and $7.0 \mathrm{~km}$ respectively. Similarly, error plots for regions of similar sizes can be given by Fig. 3.10 and Fig. 3.11 . 


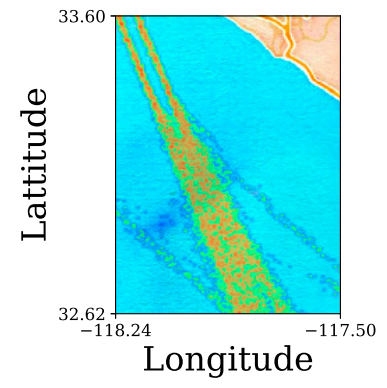

(a) Heatmap of region 3. Orange color specifies more frequent routes, while light green color specifies less frequent routes.

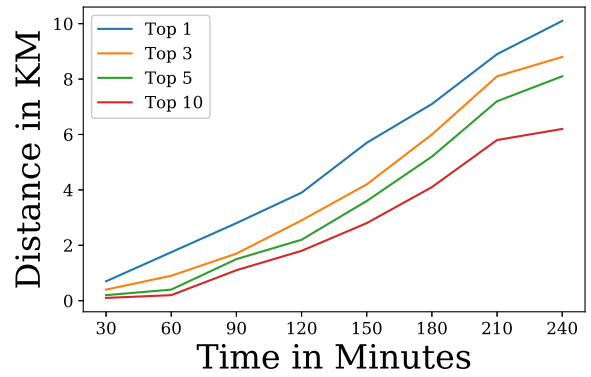

(b) Average error on testing dataset.

Figure 3.11: Performance of Markov model on region 3. 46, 558 trajectories were used to compute the transition probability. 11,640 trajectories (testing data) were used to compute the error. Top - 1 error after 4 hours is $10.2 \mathrm{~km}$.

\subsection{Limitation of Markov Model}

While the Markov model is able to compute probabilistic locations for the future, it is a computationally-expensive method. The model uses a large sized sparse matrix, the dimension of which limits the size of the region. Another limitation is that we need to save the transition probability matrix corresponding to each type, for a vessel of different types. Because of the large size of the matrix, for three timestamps, the third order Markov model is not feasible due to memory limitation. Apart from that, the model does not have the flexibility to incorporate other features, which may improve the model's performance. 


\section{Chapter 4}

\section{Traffic Prediction Using Neural Network}

\subsection{Introduction}

Neural network is a modelling technique that leverages data to learn the relationship between input data and output data. Given an input data vectors $x$ and an output data $y$, neural network helps to identify the function hypothesis $h$ for which the loss is minimum. The hypothesis $h$ is mapping from input data $x$ to predicted data $\hat{y}$ such that $\hat{y} \approx h(x)$.

A hypothesis $h$ is obtained by minimizing a loss function $\operatorname{loss}(y, \hat{y})$. For regression problems (where output values are real valued), a common loss is the mean square error loss. For the number of input sample $m$, the loss can be given as follows

$$
\operatorname{loss}(y, \hat{y})=\frac{1}{m} \sum_{i=1}^{m}\left(\hat{y}_{i}-y_{i}\right)^{2} .
$$

Neural networks are made up of small compute units called neurons [22], which are basic building blocks of large neural networks. A single neuron is parametrized by its weight vector $w$ and bias term $b$. Based on the nature of the problem, the output of a neuron is passed through activation function $a$ to get the final output. Basic neuron block can be described mathematically as follows

$$
z(x)=w^{T} x+b
$$




$$
h(x)=a(z(x))
$$

where $w \in \mathbb{R}^{n}$ is a weight vector, $x \in \mathbb{R}^{n}$ is a vector, which represents one input sample with $n$ features, and $b \in \mathbb{R}$ is a bias term. The output of a neuron is generated by first taking an inner product of a weight vector $w$ with an input vector $x$ and adding a bias term to it. This output is given to the activation function to generate the final output $h$. For regression problems, activation function $a$ is commonly taken as linear function

$$
a(x)=x
$$

For classification problems, activation function $a$ is typically taken as either sigmoid

$$
a(x)=\sigma(x)=\frac{1}{1+e^{-x}}
$$

the tanh function

$$
a(x)=\tanh (x)=\frac{e^{x}-e^{-x}}{e^{x}+e^{-x}}
$$

or the ReLu

$$
a(x)=\operatorname{Re} L u(x)=\max (0, x)
$$

Weight vector $w$ and bias term $b$ for a neuron can be learned by back propagation algorithm [22]. The problem with a single neuron is that their use is limited to data having a linear nature. To model a complex hypothesis, more than one neuron is combined to form Feed Forward Neural Network (FFNN), as shown in Fig. 4.1.

A Feed Forward Neural Network is also called a Fully Connected Network (FCN). It has three components: an input layer, one or more hidden layers and an output layer. The depth of the neural network is defined as the number of hidden layers in FCN. Each layer is characterized by weight matrices $W$, and bias vector $b$. For the 


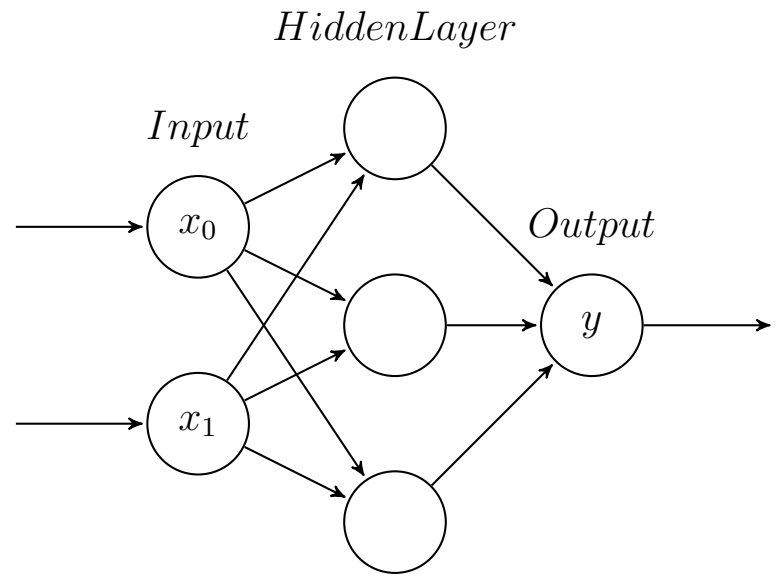

Figure 4.1: A simple example of Feed Forward Neural Network.

neural network shown in Fig. 4.1, there will be two weight matrix $W^{(0)} \in \mathbb{R}^{2 \times 3}$ and $W^{(1)} \in \mathbb{R}^{3 \times 1}$, and there will be two bias vectors $b^{(0)} \in \mathbb{R}^{3}$ and $b^{(1)} \in \mathbb{R}^{1}$.

Output is generated using a method called forward propagation [22]. Forward propagation is implemented as follows. Let activation function at the hidden layer be $a_{\text {HiddenLayer }}(x)=a^{(0)}$ and activation function at the output layer be $a_{\text {Output }}(x)=a^{(1)}$. Let the input vector be $x$,

$$
\begin{gathered}
x=\left[\begin{array}{l}
x_{0} \\
x_{1}
\end{array}\right] \\
x^{(0)}=x^{T} \\
z^{(0)}=\left(x^{(0)}\right) W^{(0)}+\left(b^{(0)}\right)^{T} \\
x^{(1)}=a^{(0)}\left(z^{(0)}\right) \\
z^{(1)}=\left(x^{(1)}\right) W^{(1)}+\left(b^{(1)}\right)^{T} \\
y=x^{(2)}=a^{(1)}\left(z^{(1)}\right)
\end{gathered}
$$

This method can be generalized to $n$ number of hidden layers. The success of neural 


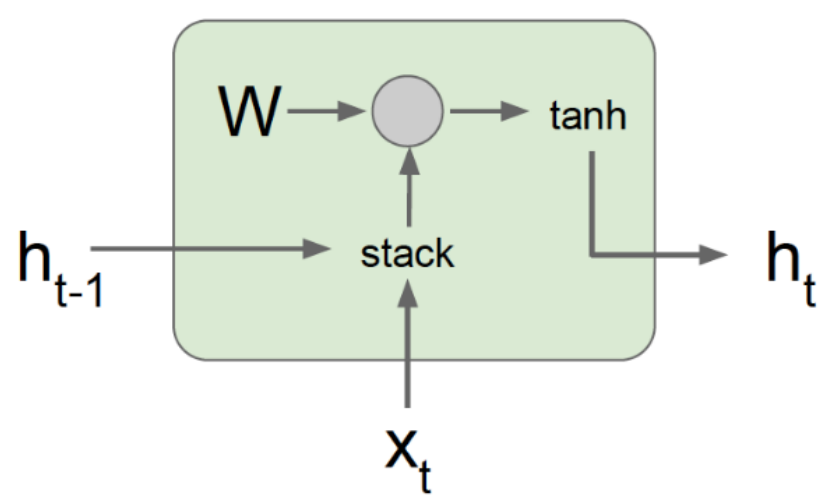

Figure 4.2: Basic unit of RNN.

network is in its ability to learn parameters of weight matrices, given enough amount of training data. Neural networks are trained using an algorithm called backpropagation 222. The backpropagation algorithm adjusts the values of weight matrices to minimize the loss between output and predicted value. Backpropagation uses optimizers like Gradient Descent (GD) [22], Stochastic Gradient Descent (SGD) [22] or Adam [23] to minimize the loss function.

\subsection{Recurrent Neural Network}

Recurrent Neural Network (RNN) is a special type of neural network which leverages sequential structure of data. While generating the output, RNN not only considers current input but also takes into account the previous output. The ability of RNN to incorporate previous output along with current input makes them suitable for the sequence prediction task.

Basic block of RNN can be described by the diagram shown in Fig. 4.2. Output of RNN can be computed as follows 


$$
\begin{gathered}
x_{\text {stacked }}=\left[\begin{array}{c}
h_{t-1} \\
x_{t}
\end{array}\right], \\
h_{t}=a\left(\left(x_{\text {stacked }}\right)^{T} W\right),
\end{gathered}
$$

where $a$ is an activation function, $h_{t-1} \in \mathbb{R}^{n}$ is an output at a previous timestamp, $n$ are the number of units of RNN. $x_{t} \in \mathbb{R}^{d}$ is an input at a current timestamp with $d$ features, $W \in \mathbb{R}^{(n+d) \times 1}$ is a weight matrix, which characterizes $\mathrm{RNN}$. The first input at a current timestamp $x_{t}$ and the output at a previous timestamp $h_{t-1}$ are stacked vertically to get $x_{\text {stacked }}$. After that $x_{\text {stacked }}$ is multiplied with $W$, output of which is given to activation function $a(x)$ to get the output at a current timestamp $h_{t}$. W is made up of two parts $W_{h h}$ and $W_{x h}$. The effect of previous outputs are incorporated by $W_{h h} \in \mathbb{R}^{n}$, and the effect of current input to the output is incorporated by $W_{x h} \in \mathbb{R}^{d}$.

Consideration of the previous output $h_{t-1}$, along with the current input $x_{t}$, gives RNN an ability to model the sequential structure of the data. While generating the output using multiple timestamps, the same weight matrix $W$ is used, because of which RNN suffers from the problem of vanishing gradient or exploding gradient while training, which makes RNN difficult to train.

\subsection{Long-Short Term Neural Network}

Long-Short Term Neural Network (LSTM) is a special type of RNN with additional gates to solve the problem of vanishing gradient and exploding gradient, which makes them suitable for the sequence prediction task.

The basic block of LSTM can be described by the diagram shown in Fig. 4.3. Four additional gates are included with the basic block of RNN so that learning sequential structure becomes easier. The memory or state of the basic block is characterised by $c_{t}$. 


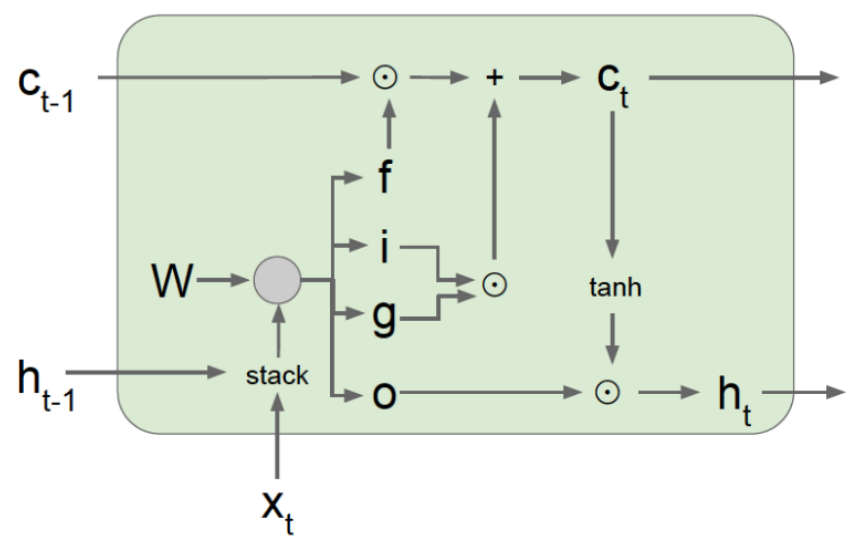

Figure 4.3: Basic unit of LSTM.

(Hochreiter et al. 1997)

How much information should be incorporated from the current input to the current state is controlled by input gate $i$. How much information should be incorporated from the previous memory or previous state to the current state is controlled by forget gate $f$. How to update the current state is controlled by $g$. How much information should be used from the current state $c_{t}$, the previous output $h_{t-1}$, and the current input $x_{t}$ to the output is controlled by the output gate $o$. Output of LSTM can be computed as follows

$$
\begin{gathered}
i=\sigma\left(W_{i} h_{t-1}+U_{i} x_{t}+b_{i}\right), \\
o=\sigma\left(W_{o} h_{t-1}+U_{o} x_{t}+b_{o}\right), \\
f=\sigma\left(W_{f} h_{t-1}+U_{f} x_{t}+b_{f}\right), \\
g=\tanh \left(W_{g} h_{t-1}+U_{g} x_{t}+b_{g}\right),
\end{gathered}
$$

where $W_{i}, W_{o}, W_{f}, W_{g} \in \mathbb{R}^{n \times n}$, bias terms $b_{i}, b_{o}, b_{f}, b_{g} \in \mathbb{R}^{n}$, and $U_{i}, U_{o}, U_{f}$, $U_{g} \in \mathbb{R}^{n \times d}, n$ is number of units of LSTM, and $d$ is number of features in input $x_{t}$.

$$
h_{t}=o \odot \tanh \left(c_{t}\right)
$$




$$
c_{t}=f \odot c_{t-1}+i \odot g
$$

where $\odot$ represents element wise multiplication. First $i, o, f$, and $g$ are computed using current input $x_{t} \in \mathbb{R}^{d}$ and previous output $h_{t-1} \in \mathbb{R}^{n}$. Output $h_{t} \in \mathbb{R}^{n}$ is computed by element wise multiplication of $o$ and $\tanh \left(c_{t}\right)$. Similarly the cell state is updated using $f, g$ and $i$ with $c_{t-1}$.

Additional gates in LSTM give it flexibility to learn long term dependency in the sequential data. LSTM solves the problem of vanishing gradient and exploding gradient at the expense of complex architecture, which increases the training time for the LSTM-based neural network.

\subsection{Ship Traffic Prediction with Neural Networks}

The problem of vessel trajectory prediction can be formulated as a time series forecasting problem because data is assumed to be sequential in nature. The task is to predict future locations of the vessel based on its current and previous locations. Future location coordinates of the vessel can be assumed to be generated from a random

distribution, whose parameters are dependent upon current and previous locations. Suppose location of vessel at time $t$ is given as $x_{t}$, where

$$
x_{t}=\left[\begin{array}{c}
\text { Longitude }_{t} \\
\text { Latitude }_{t}
\end{array}\right] \text {. }
$$

Based on this convention, the next location of the vessel can be denoted by $x_{t+1}$. Similarly previous locations can be denoted by $x_{t-1}, x_{t-2}, x_{t-3}, x_{t-4}, \ldots$ and so on. The problem is equivalent to computing the value of $x_{t+1}$ given $x_{t}, x_{t-1}, x_{t-2}, \ldots$ and so on. To predict $x_{t+1}$ from historical data, we wish to learn a hypothesis $h$ such that 


$$
x_{t+1} \approx h\left(x_{t}, x_{t-1}, \ldots, x_{t-k}\right),
$$

where $k$ is the number of previous timestamps. Due to the sequential nature of the data, the hypothesis $h$ in this case is best modelled by LSTM. Finding hypothesis $h$, which approximates the relation between $x_{t+1}$ and $\left(x_{t}, x_{t-1}, \ldots, x_{t-k}\right)$, is equivalent to the learning parameters of the LSTM-based network. The architecture used in this work is given by Fig. 4.4. Our model architecture is inspired by [1]. The number of units on each layer is selected based on the model architecture described in [1]. There are two ways to incorporate inputs. Either they can be incorporated as time series inputs, or they can be incorporated as fusion inputs. Based on these, there are two parts of the network, the LSTM part (right side), and the Fusion part (left part). The LSTM part of the neural network is made up of the first LSTM layer, which has 50 units of LSTM as in Fig. 4.3. The first layer of LSTM is followed by the second layer of LSTM, which also has 50 units. The output of the second layer of LSTM is given to the Fully Connected Layer 1 with 150 neurons. The activation function used for the Fully Connected Layer 1 is ReLu, and the output of the Fully Connected Layer 1 is given to Fully Connected Layer 2, which is passed through the activation function to get the final output. The Fusion part of the neural network is made up of Fully Connected Layer 3, which has 150 neurons, the output of which is concatenated with Fully Connected Layer 2. LSTM layers 1 and 2 are used to learn the sequential structure in the data. Fully Connected Layers 1 and 2 are used to map the output generated by the LSTM layers to actual coordinates of Latitude and Longitude. 


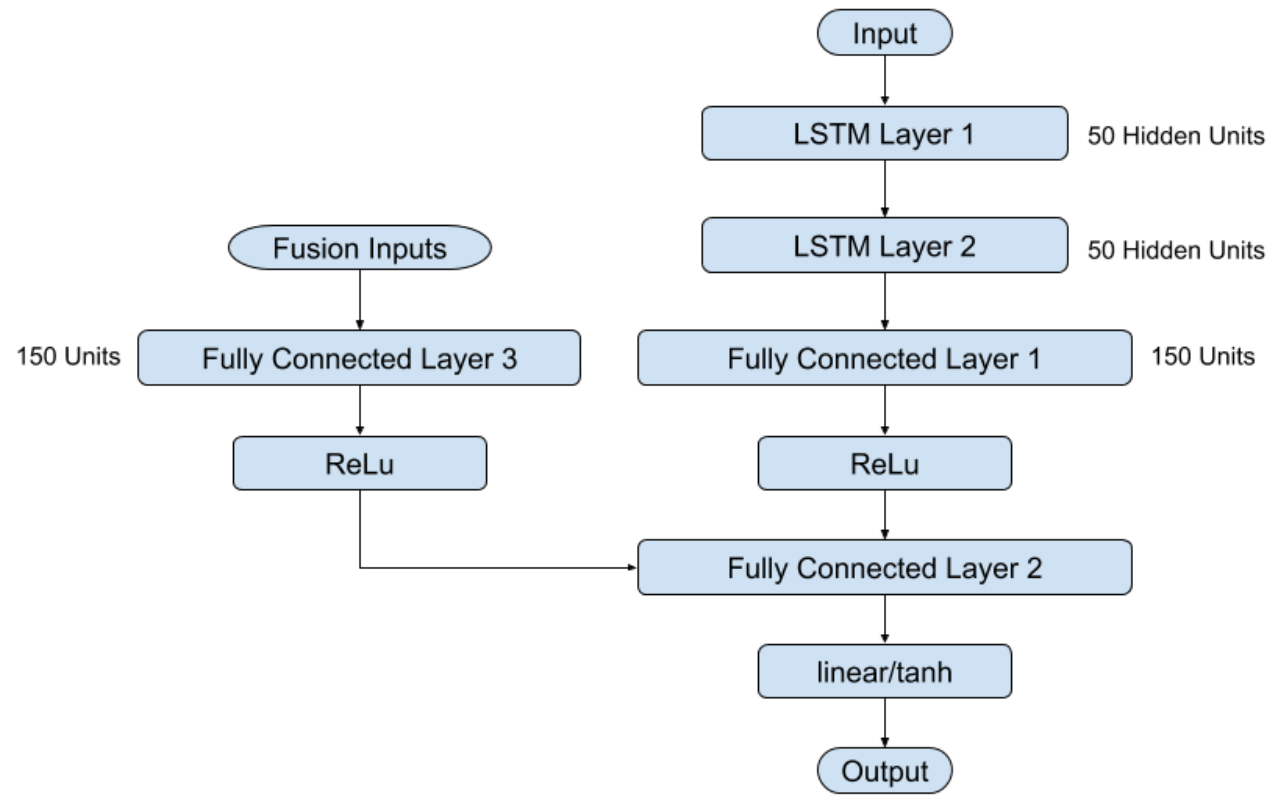

Figure 4.4: Architecture of proposed model. Number of units in each layer are selected according to [1.

\subsection{Experimental Setup}

After pre-processing the data using the steps described in Section 2.3, the learning problem is formulated as follows. Feature space or input vector is made up of the vessel's current and previous location and the output vector is made up of the next location or future location of the vessel. We trained our LSTM based model with different input feature spaces to develop the final approach. An Adam optimizer with a learning rate of 0.001 was used to train the model. For development, training, and prediction, the Keras 24 library was used. The models were trained for 1,000 iterations, with a batch size of 4,096 samples. 


\subsubsection{Error Metric}

To compare different architecture/models' performance, we have defined the error metric, which considers the haversine distance between the predicted location and actual location. This error metric is useful for assesing the performance of different combinations of architecture/model and input features. All the combinations would give Latitude and Longitude coordinates corresponding to future time stamps for the prediction task. The time difference between consecutive timestamps is 30 minutes. The prediction error for one trajectory is defined as the haversine distance between actual position coordinates and predicted position coordinates. For one trajectory, We have computed predictions for 8 timestamps, i.e., predictions from 30 minutes to 4 hours in the future. Corresponding to every 30 minute prediction, there is an error which is equal to the haversine distance between the actual and predicted location coordinates

$$
e_{i}^{t+n T}=2 R \sin ^{-1}\left(\sqrt{\sin ^{2}\left(\frac{L^{2} t_{2}-L a t_{1}}{2}\right)+\cos \left(L a t_{2}\right) \cos \left(L a t_{1}\right) \sin ^{2}\left(\frac{\operatorname{Lon}_{2}-\operatorname{Lon}_{1}}{2}\right)}\right),
$$

where

$$
\begin{gathered}
n=\{1,2,3,4,5,6,7,8\}, \\
T=30 \mathrm{~min} .
\end{gathered}
$$

Generalizing this idea to all the trajectories in the testing dataset, and taking the average of all those errors, is the error metric, which has been used here to evaluate the performance of different approaches

$$
e_{\text {total }}^{t+n T}=\frac{1}{m} \sum_{i=1}^{m} e_{i}^{t+n T}
$$




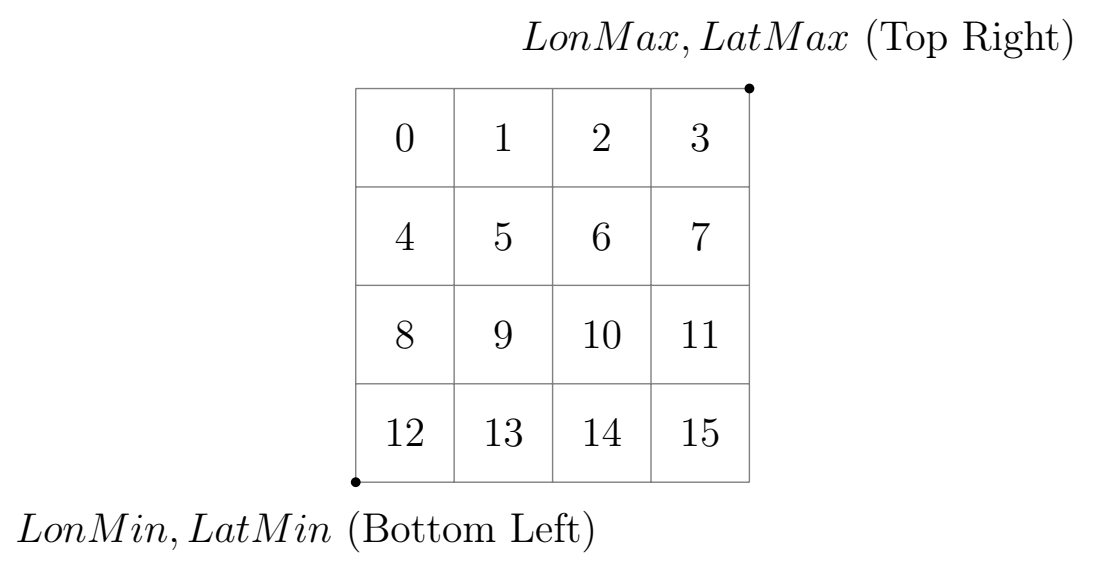

Figure 4.5: Numbered representation of ROI with numbers assigned to each cell.

where $m$ is equal to the number of trajectories in the testing data.

\subsubsection{Predicting Classes/Cell Number Corresponding to Future Location}

Following the approach used in the second-order Markov model, feature space or an input vector was made up of cell numbers corresponding to two locations, the current location and the previous location, i.e., the location half an hour earlier. The output vector was made up of the cell number corresponding to the next location of the vessel, i.e. the location of the vessel after half an hour.

The region was divided into the grid as in Fig. 3.2, then according to the vessel's position in any of the cells, the number of that cell was taken as a feature. These input values were then transformed into one hot encoded vector representation. The width of the one hot encoding was equal to the total number of cells. For Fig. 4.5, one hot encoding would have a length of 16 . Using this encoding, we require a length-16 vector corresponding to the previous location and a length- 16 vector corresponding to the next location, yielding a length-32 feature vector. Similarly, the output vector also would be of length-16, which would assign probability values to all 16 elements, with the maximum value of probability assigned to a number, where the next location would be most probable. The center point of that cell would be used as a predicted 


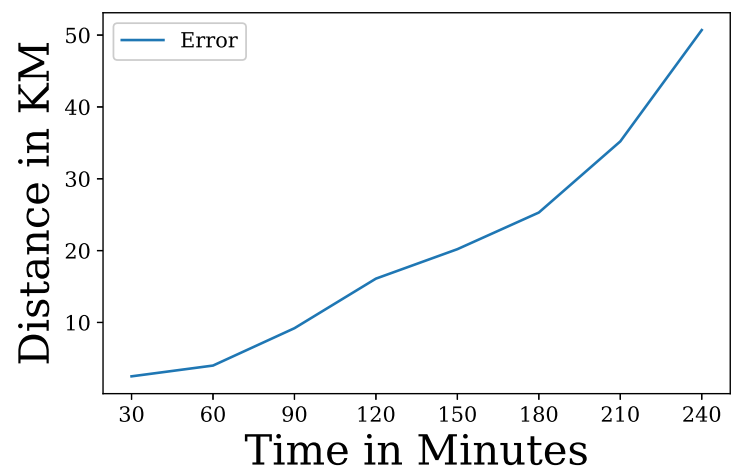

Figure 4.6: Error plot for cell prediction. The model was not able to learn the underlying distribution giving a large value of errors.

value.

We pre-processed the data for the region characterized by

1. $L O N_{-} M I N=-120.50$,

2. $L O N_{-} M A X=-119.00$,

3. $L A T T_{-} M I N=33.90$,

4. $L A T_{-} M A X=34.44$.

with a grid size of $0.01 \times 0.01$. For this region, we had a total of 8,100 cells. So the input vector was of size 16,200 , and the output vector was of size 8,100 . We used this data to train the LSTM part of the neural network described in figure 4.4. Because of the classification nature of the problem, tanh activation was used after Fully Connected Layer 2. The loss used for training purposes was cross entropy loss. There were 53, 234 trajectories for training and 14, 201 for testing. After training the model for 1,000 iterations, the error plot can be described by figure 4.6 .

The problem with this approach was that the model could not learn the underlying distribution because of the large number of classes to predict from. Another problem 
was that this model could not take into account geographic closeness of the misclassified cell and the actual cell. This model was penalizing all the errors in a similar way.

\subsubsection{Predicting Continuous Value of Longitude and Latitude}

To overcome the problem of a large number of output classes to predict, instead of transforming the inputs and outputs to classes as described in the previous section, we used continuous values of inputs as feature space, i.e, $x_{t-1}$ and $x_{t}$ along with other features such as length, destination, and type of the vessel. The next location, i.e., $x_{t+1}$, was used as an output label. Instead of classification, the problem was formulated as a regression problem. The following subsections describe the implementation and performance of different combinations of input features and neural network architecture. The region used for these different implementations was the same as in the previous section. Similarly training and testing data were also the same.

\section{Using Position Coordinates of Two Timestamps}

In this approach, we used the LSTM part of the neural network, as described in Fig. 4.4. Because of the continuous value of the output, linear activation was used at the output layer. Feature space, or input to the neural network, was made up of position coordinates at the current time, and position coordinates at the time half an hour before. Current position $x_{t}$ and previous position $x_{t-T}$ have been used to predict $x_{t+T}$. We refer to this implementation as Vanilla implementation. Error on testing data is shown in Fig. 4.7

\section{Using Position Coordinates of Two Timestamps and Type of Vessel}

In this approach, we used the type of vessel as a feature along with the locations of two timestamps. The idea behind using type as a feature was based on the assumption 


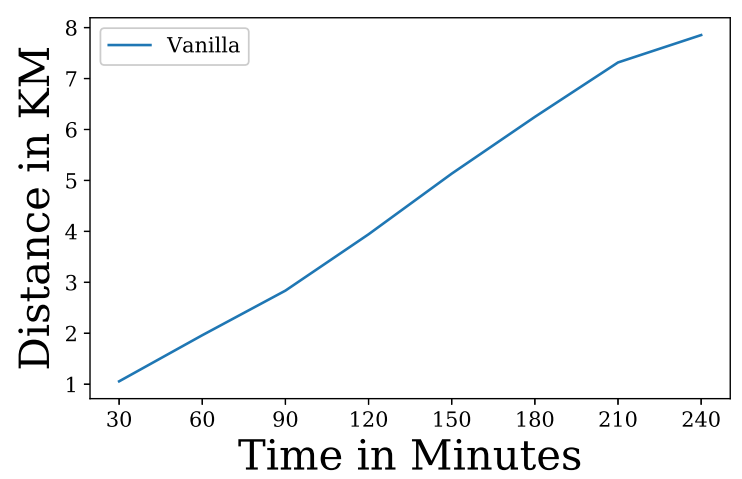

Figure 4.7: Error plot for Vanilla LSTM. Error after 4 hours is $7.9 \mathrm{~km}$.

that vessels of one type would behave in a similar way. We have used data for two kinds of vessels.

\section{Cargo}

\section{Tanker}

The type feature was encoded as a vector of length two, based on the vessel type of the trajectory data. For the tanker vessels, it was encoded as $[1,0]$, and for cargo, it was encoded as $[0,1]$. There were two ways to incorporate this feature: along the LSTM part of the neural network shown in Fig. 4.4, or along the fusion part of the neural network shown in Fig.4.4. We trained both variations of the network. After observing the error plot in Fig. 4.8, we came to the conclusion that adding type as a feature improves the performance of the model. Error after 4 hours drops by $16.37 \%$ on the addition of type as a feature along the LSTM part. On the other hand, performance of network becomes worse on the addition of type as a feature along the Fusion part. 


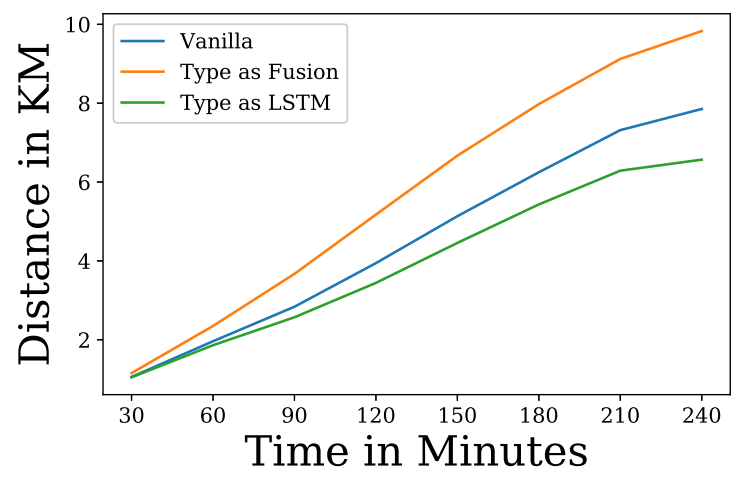

Figure 4.8: Comparison error plot on addition of type as a feature. Addition of type as a time series input improves the performance of the model. Error after 4 hours is $6.6 \mathrm{~km}$.

\section{Using Position Coordinates of Two Timestamps and Type of Vessel and Length of Vessel}

In this approach, along with the type of vessel, we tried to incorporate the physical dimensions of the vessel. The assumption behind considering the physical dimensions was that physical dimensions like the length of the vessel would be helpful for incorporating the dynamics of the vessel. We trained the neural network shown in Fig. 4.4 for both combinations, i.e. length as Fusion input or length as LSTM input. After training the neural networks, the networks' performance on the testing set can be summarized by Fig. 4.9. After analyzing the plots, we came to the conclusion that the addition of length as a feature does not help to improve performance, whether length gets added as Fusion input or LSTM input.

\section{Using Position Coordinates of Two Timestamps and Type of Vessel and Destination of Vessel}

In this approach, along with previous position coordinates and type of vessel, we incorporated the destination of the trajectory as a feature. Destination as a feature was not available in the dataset. To add destination as a feature, we developed a 


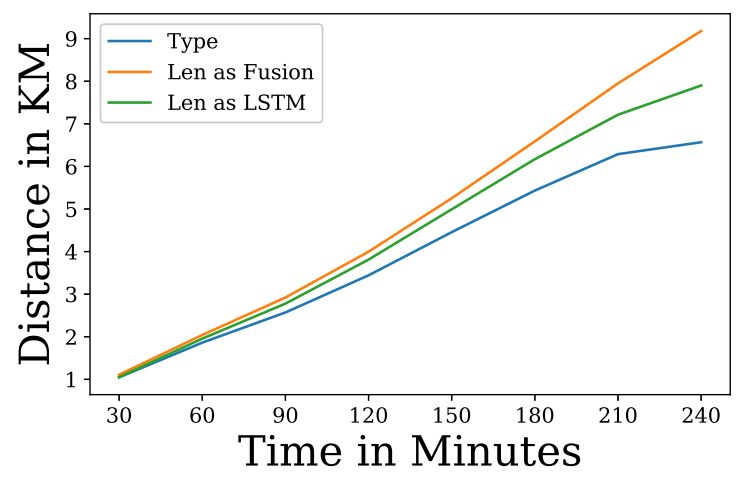

Figure 4.9: Comparison error plot on addition of length as a feature. Addition of length does not help to improve the performance.

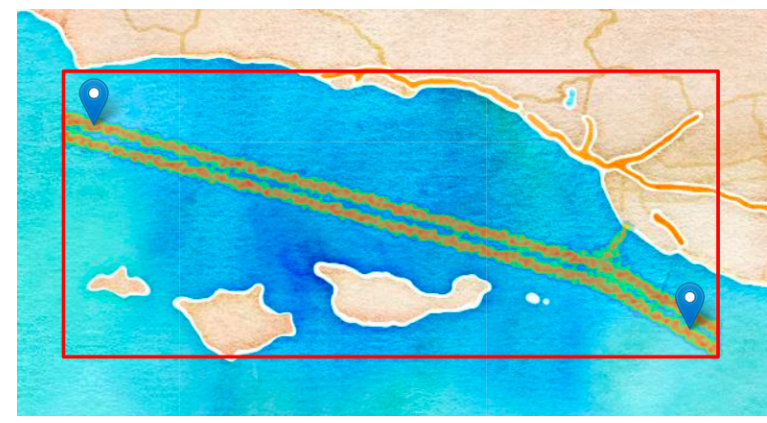

Figure 4.10: Destination assignment using clustering.

scheme similar to [5]. For all the trajectories, the last point of the trajectory was taken; after that, all these points were given to a clustering algorithm called KMeans [9], which assigned a cluster to every destination point. Based on the cluster assignment, the cluster center's coordinates were taken as destination features for that trajectory. For the region, the clustering algorithm's output gave two destinations, which are highlighted in Fig. 4.10.

After assigning a destination to each trajectory, the feature vector was made up of the position coordinates of the previous two timestamps, type of the vessel, and Latitude and Longitude coordinates of the destination. We trained the neural network shown in Fig. 4.4 for both combinations, i.e., DestinationLatitude and DestinationLongitude as Fusion inputs or DestinationLatitude and DestinationLongitude 


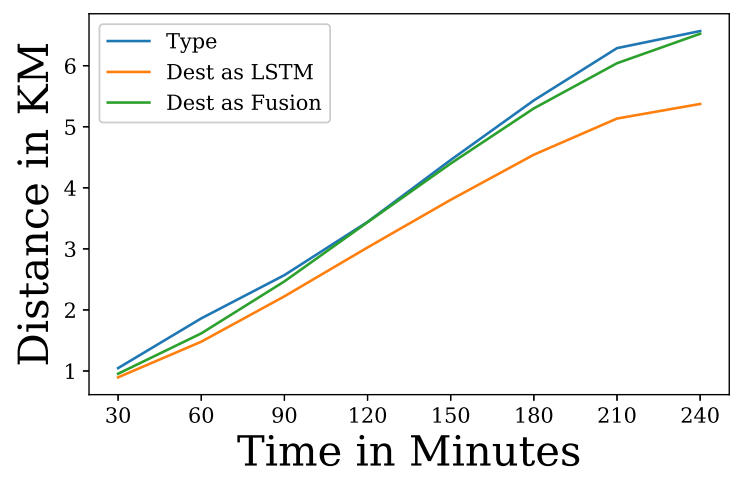

Figure 4.11: Comparison error plot on addition of destination as a feature. Performance of model improves on addition of destination as a time series input. Average error after 4 hours drops to 5.4 $\mathrm{km}$.

as LSTM inputs. After training the neural networks, the performance on the testing set can be summarized by Fig. 4.11.

The addition of destination as a feature improved the performance of the model if the destination was incorporated as an LSTM input. The performance of the model did not change much on the addition of the destination as a Fusion input.

\section{Using Position Coordinates of Three Timestamps}

In this approach, instead of using the position coordinates of two timestamps, we incorporated three timestamp's position coordinates, i.e, the feature space consists of the position coordinate of current time $x_{t}$, position coordinates at time $t-T x_{t-T}$, and position coordinates at time $t-2 T x_{t-2 T}$. After training the neural network represented shown in Fig. 4.4, We computed the error, which is shown in Fig. 4.12, On comparison of the error with the model, which had feature space consisting of the position of two timestamps, we concluded that feature space consisting of three timestamps has helped to improve the performance of the model at the cost of longer training time. We also tried position coordinates of four timestamps, but the performance gain was not significant at the cost of longer training time. 


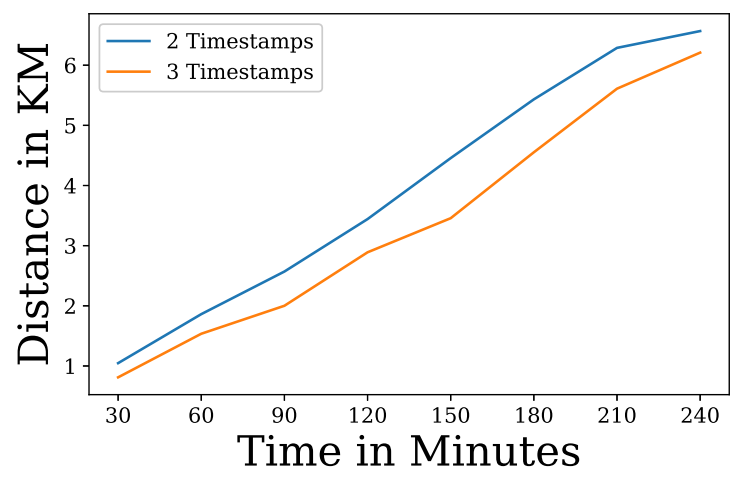

Figure 4.12: Comparison error plot of two and three timestamps. Position coordinate of one more timestamp helps to improve the performace of the model.

\section{Performance On Other Regions of Similar Size}

After trying different combinations of input features, we trained the model using data from different regions of similar sizes and evaluated their performances on testing data corresponding to those regions to verify the generability of the method on different regions. Performance of the model on different regions of similar sizes can be given by Fig. 4.13 and Fig. 4.14 

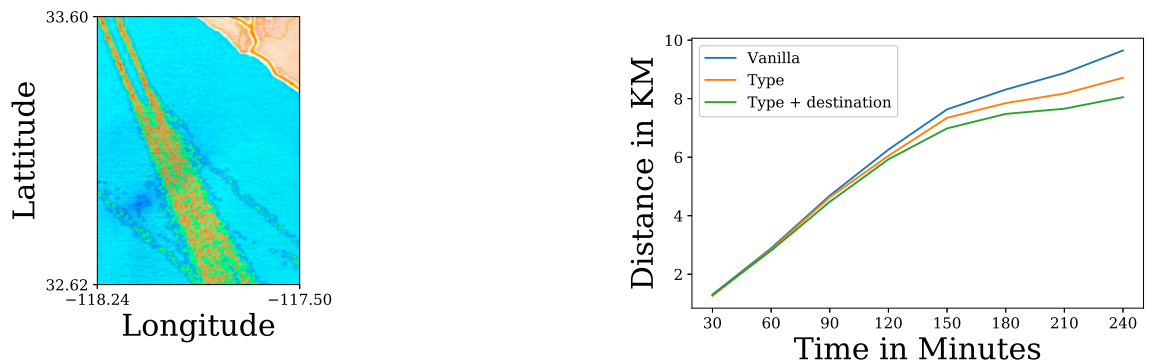

(a) Heatmap of region 2. Orange color specifies more frequent routes, while light green color specifies less frequent routes.

(b) Comparison error plots for different input combination.

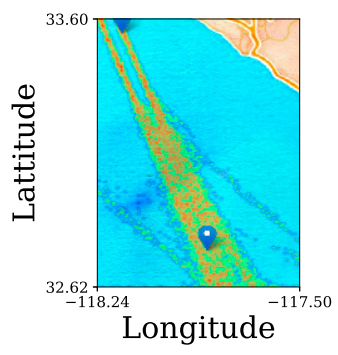

(c) Heatmap of region 2 with destination. Orange color specifies more frequent routes, while light green color specifies less frequent routes. Destinations are marked

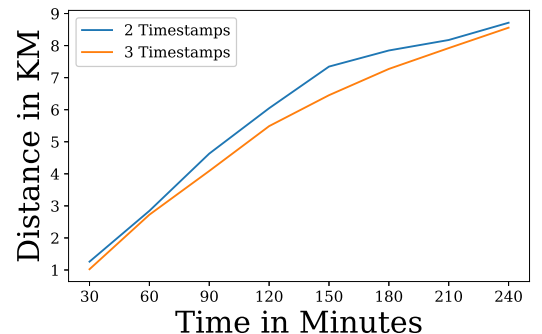
by blue markers.

Figure 4.13: Error plots for Region 2. The model's performance with type and destination as a feature is better compared to the Vanilla implementation. The model with position coordinates of three timestamps performs better than the position coordinates of two timestamps. 

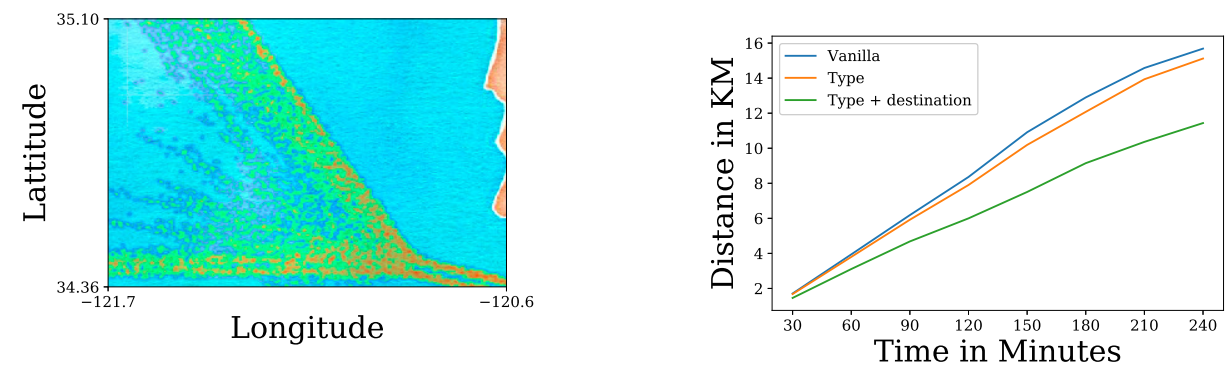

(a) Heatmap of region 3. Orange color specifies more frequent routes, while light green color specifies less frequent routes.

(b) Comparison error plots for different input combination.

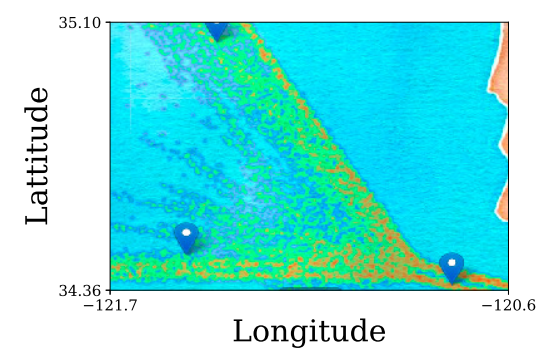

(c) Heatmap of region With Destination. Orange color specifies more frequent routes, while light green color specifies less frequent routes. Destinations are marked

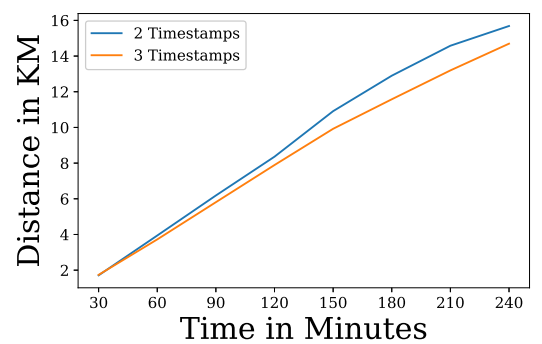

(d) Comparison error plots for 2 and 3 by blue markers.

Figure 4.14: Error plots for Region 3. The model's performance with type and destination as a feature is better compared to the Vanilla implementation. Effect of destination is prominent because of diverging lanes. The model with position coordinates of three timestamps performs better than the position coordinates of two timestamps. 


\subsubsection{Combining All These for A Bigger Region With More Data}

One advantage of the neural network-based method is that it is scalable for the bigger region as well, unlike the Markov model. We extended the region of interest to the following coordinates,

1. LON_MIN $=-122.00$,

2. $L O N_{-} M A X=-119.50$,

3. $L A T \_M I N=34.00$,

4. LAT_MAX $=36.00$.

which is a much bigger region than the previously considered regions. The smaller region had an approximate area of $22,500 \mathrm{~km}^{2}$, compared to the bigger region that had an approximate area of 101,124 $\mathrm{km}^{2}$. Along with the bigger region, we also took the data from the years 2015 to 2017. After pre-processing the data described by Section 2.1, the number of training trajectories came out to be 189,923 , and the number of testing trajectories came out to be 47,482 . Based on the assumption that there can be some seasonal patterns, along with the location coordinates of three timestamps, the type of the vessel, and the destination of the vessel, we also incorporated the month of trajectory as a feature. The hypothesis, which we were trying to learn can be described by

$$
x_{t+T}=h\left(x_{t}, x_{t-T}, x_{t-2 T}, \text { Type, Destination, Month }\right)
$$

As the region got bigger, the clustering based destination scheme was not effective, so we took the last coordinates of the trajectory as the destination. We trained the LSTM part of the neural network shown in Fig. 4.4 and trained the model. The performance of the model on testing trajectory is shown in the Fig. 4.15 


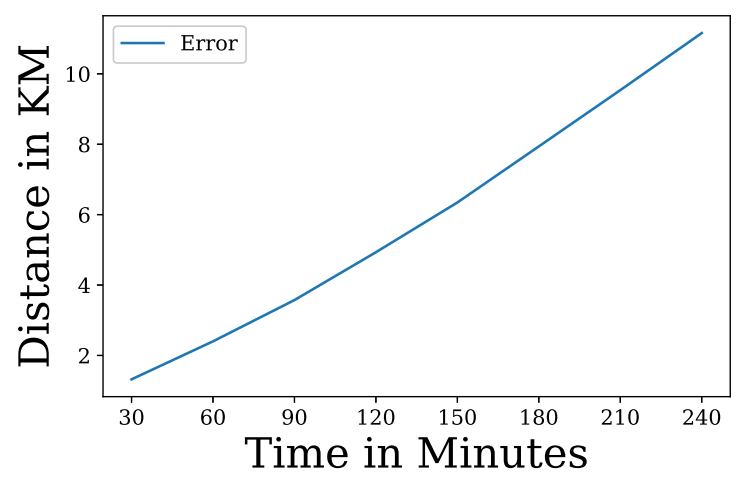

Figure 4.15: Error on bigger region. Averge error for 30 minute prediction is $1.1 \mathrm{~km}$. Average error after 4 hours is $11.2 \mathrm{~km}$.

\section{Taking regional SOG and COG into consideration}

After carefully analyzing trajectories that gave a higher error, we found out that trajectories with irregular values of Speed Over Ground (SOG) and Course Over Ground (COG) were responsible for higher error. Motivation for incorporating SOG and COG is that there can be few regions where vessels may move faster than average or slower than average. SOG corresponds to the speed of the vessel and COG corresponds to the direction of the vessel. Adding this information may help the model to learn the underlying distribution in a better way. To incorporate SOG and COG, we used historical data to compute the median value of SOG and COG for each cell of the grid. These grid dependent SOG and COG values were used as additional features to help the model incorporate region specific side information. In this case, our features utilized are $x_{t}, x_{t-T}$, $x_{t-2 T}$, Type, Destination, Month, MedianSOG $G_{x_{t}}, M e d i a n C O G_{x_{t}}, M e d i a n S O G_{x_{t-T}}$, MedianCOG ${ }_{x_{t-T}}$, MedianSOG $G_{x_{t-2 T}}$, MedianCOG $G_{x_{t-2 T}}$. MedianSOG $_{x_{t}}$ and MedianCOG $G_{x_{t}}$ were values of median SOG and COG for a cell corresponding to $x_{t}$. We trained the model and computed its performance on a testing dataset, which is given by Fig. 4.16 .

After that, we trained the model for the much bigger region, one on the west coast and one near the Gulf of Mexico, the performance of the model on both the regions 


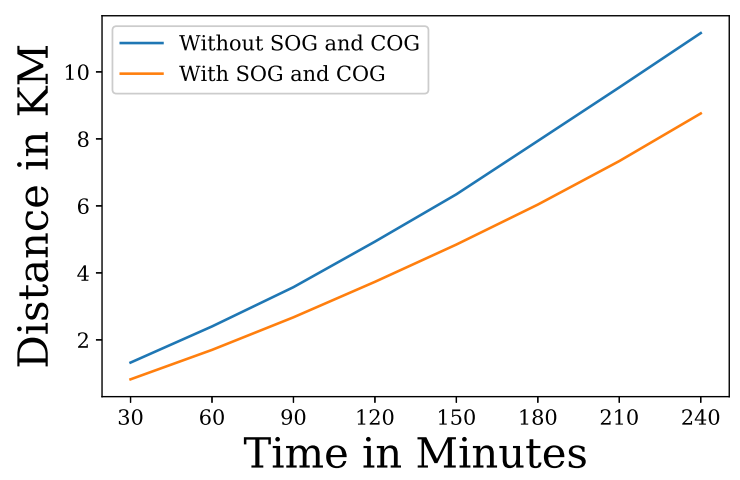

Figure 4.16: Error comparison on addition of regional SOG and COG. Addition of SOG and COG as a feature improves the peroformance of the model. The average after 4 hours drops to $8.2 \mathrm{~km}$.
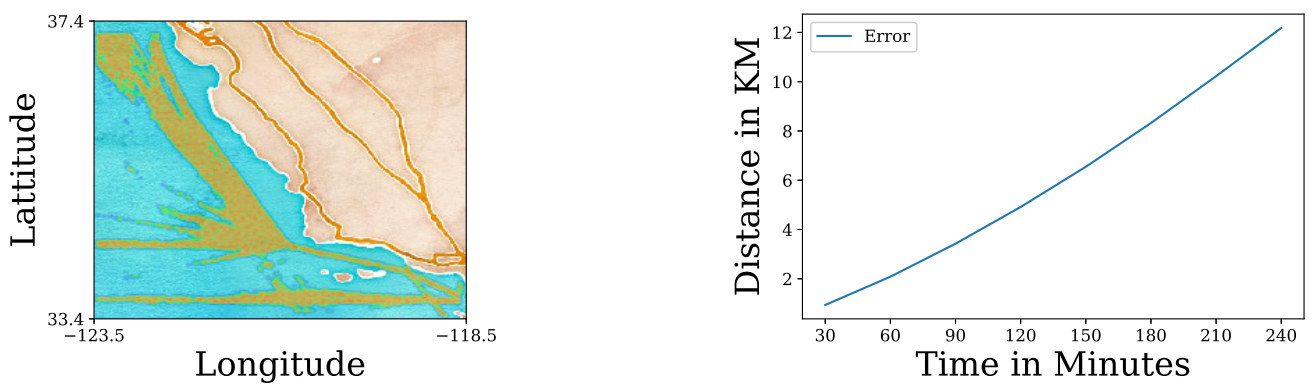

Figure 4.17: Error plot for bigger region near west coast. Orange color specifies more frequent routes, while light green color specifies less frequent routes. Average error after 4 hours is $12.18 \mathrm{~km}$. A total of 362, 597 trajectories were used for the training, and 90,650 trajectories were used for the testing.

can be given by Fig. 4.17 and Fig. 4.18 .

\subsection{Comparison with Markov Model}

For the region used in Section 4.5.2, we have used the same trajectory data to compute the transition probability matrix to train the LSTM-based model for direct comparison. The comparison between Markov model and LSTM-based model is shown in Fig. 4.19. Fig. 4.19 shows comparison of Top - 1 error generated by second-order Markov model and LSTM-based model, which uses the position of two timestamps and type of the vessel as a feature. From the figure, we can conclude that LSTM-based model performs better than second-order Markov model due to its ability to learn complex distribution 

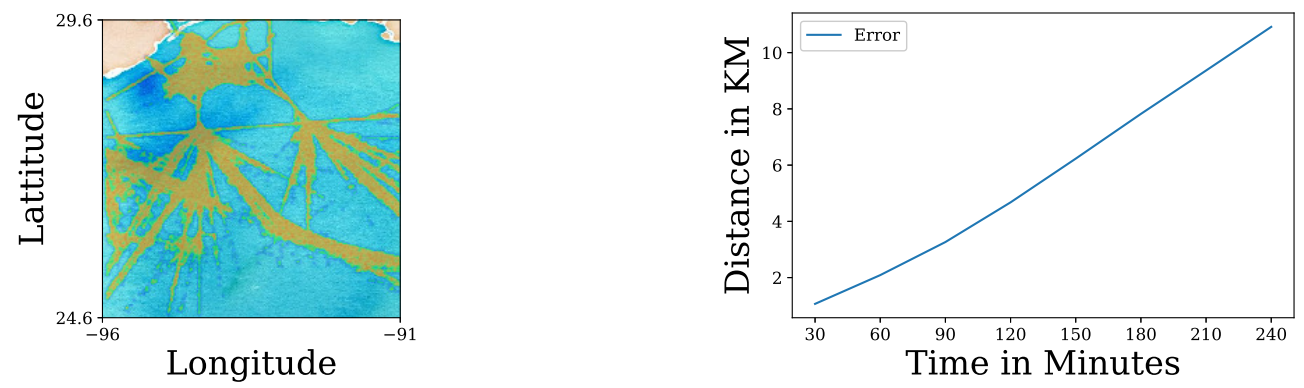

Figure 4.18: Error plot for bigger region near Gulf. Orange color specifies more frequent routes, while light green color specifies less frequent routes. Average error after 4 hours is $10.91 \mathrm{~km}$. A total of 1,436, 023 trajectories were used for the training, and 359, 006 trajectories were used for the testing. Due to large number of training trajectories average error is less than the west coast region.

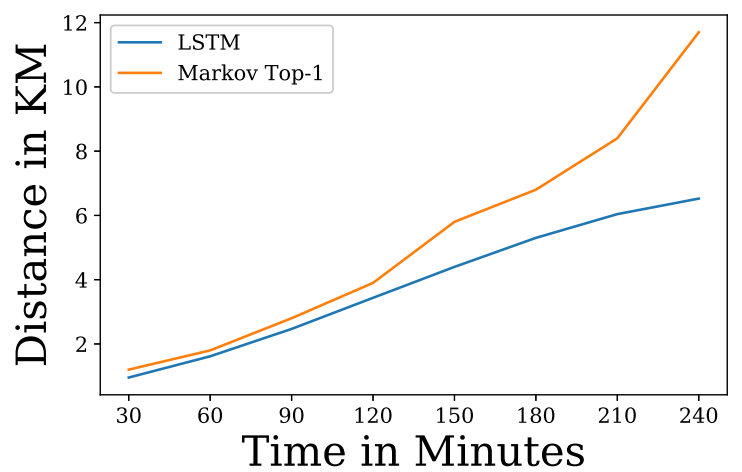

Figure 4.19: Error comparison of Markov model and LSTM based model. The Top -1 error for the Markov model increases faster compared to LSTM based model for the long term prediction.

from the data.

\subsection{Quantitative Results}

The average error for the prediction of 30 minutes for a region specified in Section 4.5.4 near the west coast is $820 \mathrm{~m}$, which is comparable to the error given in [1, i.e., $670 \mathrm{~m}$. Similarly, error at an interval of 90 minutes is $2.6 \mathrm{~km}$, which is comparable to $2.8 \mathrm{~km}$ given in [1] for 100 minute prediction. For the prediction of 4 hours, the same region has an error of $8.2 \mathrm{~km}$. The average error for the prediction of 30 minutes for a bigger region $\left(407,044 \mathrm{~km}^{2}\right)$ near the west coast is $930 \mathrm{~m}$. For the prediction of 4 hours, the same region has an error of $12.18 \mathrm{~km}$. Error values for the west coast can 
be summarized by the following table.

Table 4.1: Error for bigger region near west coast

\begin{tabular}{|c|c|}
\hline Time in minutes & Mean error in km \\
\hline 30 & 0.93 \\
60 & 2.08 \\
90 & 3.42 \\
120 & 4.9 \\
150 & 6.5 \\
180 & 8.3 \\
210 & 10.2 \\
240 & 12.18 \\
\hline
\end{tabular}

Similarly, the average error for the bigger region near the Gulf of Mexico after an interval of 30 minutes is $1.1 \mathrm{~km}$. The following table summarizes error values for the region near the Gulf of Mexico.

Table 4.2: Error for bigger region near Gulf of Mexico

\begin{tabular}{|c|c|}
\hline Time in minutes & Mean error in $\mathrm{km}$ \\
\hline 30 & 1.06 \\
60 & 2.08 \\
90 & 3.26 \\
120 & 4.6 \\
150 & 6.2 \\
180 & 7.8 \\
210 & 9.3 \\
240 & 10.91 \\
\hline
\end{tabular}




\section{Chapter 5}

\section{Conclusion \& Future Work}

This thesis has presented two methods to solve the problem of vessel trajectory prediction. We have presented a second-order Markov model, which can be used for regions of smaller sizes $\left(22,500 \mathrm{~km}^{2}\right)$ to predict the vessel's location. We have implemented an LSTM-based neural network model, which is not limited by the size of the region and also takes into consideration other parameters to improve the accuracy of the prediction.

\subsection{Markov Model}

The second-order Markov model can be used to predict the vessel's location probabilistically for a small region. We have presented a method, which can be used to compute the transition probability matrix for the second-order Markov model. Once the computation of the transition probability matrix is done, it can be used with the vessel's current and previous locations to make the prediction. We showed the results of this method on different regions near the coasts of the United States, to verify its generability. The average error after 4 hours of prediction is $11.7 \mathrm{~km}$.

\subsection{Neural Network-Based Model}

We have implemented an LSTM-based neural network model to predict the location of the vessels. We tried different combinations of the feature space to come up with the 
final implementation of the model. After comparing the performance of second-order Markov model and LSTM-based neural network model on a data of the same region, we found that LSTM-based method outperforms the second-order Markov model. The average error on same region as Markov model is $5.4 \mathrm{~km}$ for LSTM-based neural network. For the region of size 407, $044 \mathrm{~km}^{2}$ near the west coast, the average error after 4 hours of prediction is $12.18 \mathrm{~km}$.

In this thesis, we have developed a model specific to two types of vessels. Applicability of the model to ships of other types should be investigated. Apart from that, complex network architecture and a combination of both methods need to be investigated to improve the performance of the model. Surprisingly, the addition of inputs along the Fusion layer has not helped to improve the performance, which can be investigated further. We have provided a prediction error of up to 4 hours. The performance of a model for prediction of more than 4 hours can be proposed as a future work. 


\section{Bibliography}

[1] S. P. Liraz, "Ships' trajectories prediction using recurrent neural networks based on AIS data," Naval Postgraduate School Monterey United States, Tech. Rep., 2018.

[2] L. P. Perera, P. Oliveira, and C. G. Soares, "Maritime traffic monitoring based on vessel detection, tracking, state estimation, and trajectory prediction," IEEE Transactions on Intelligent Transportation Systems, vol. 13, no. 3, pp. 1188-1200, 2012.

[3] F. Mazzarella, V. F. Arguedas, and M. Vespe, "Knowledge-based vessel position prediction using historical AIS data," in 2015 Sensor Data Fusion: Trends, Solutions, Applications (SDF). IEEE, 2015, pp. 1-6.

[4] G. Pallotta, S. Horn, P. Braca, and K. Bryan, "Context-enhanced vessel prediction based on Ornstein-Uhlenbeck processes using historical ais traffic patterns: Realworld experimental results," in 17th international conference on information fusion (FUSION). IEEE, 2014, pp. 1-7.

[5] G. Pallotta, M. Vespe, and K. Bryan, "Vessel pattern knowledge discovery from AIS data: A framework for anomaly detection and route prediction," Entropy, vol. 15, no. 6, pp. 2218-2245, 2013.

[6] P. Coscia, P. Braca, L. M. Millefiori, F. A. Palmieri, and P. Willett, "Multiple Ornstein-Uhlenbeck processes for maritime traffic graph representation," IEEE 
Transactions on Aerospace and Electronic Systems, vol. 54, no. 5, pp. 2158-2170, 2018 .

[7] P.-R. Lei, T.-H. Tsai, and W.-C. Peng, "Discovering maritime traffic route from AIS network," in 2016 18th Asia-Pacific Network Operations and Management Symposium (APNOMS). IEEE, 2016, pp. 1-6.

[8] M. Ester, H.-P. Kriegel, J. Sander, X. Xu et al., "A density-based algorithm for discovering clusters in large spatial databases with noise." in Proc.2nd Int. Conf. Knowledge Discovery and Data Mining (KDD'96), 1996, pp. 226-231.

[9] S. Shalev-Shwartz and S. Ben-David, Understanding machine learning: From theory to algorithms. Cambridge university press, 2014.

[10] G. E. Uhlenbeck and L. S. Ornstein, "On the theory of the Brownian motion," Physical review, vol. 36, no. 5, p. 823, 1930.

[11] S. Hexeberg, A. L. Flåten, E. F. Brekke et al., "AIS-based vessel trajectory prediction," in 2017 20th International Conference on Information Fusion (Fusion). IEEE, 2017, pp. 1-8.

[12] G. Van Brummelen, Heavenly mathematics: The forgotten art of spherical trigonometry. Princeton University Press, Princeton, 2012.

[13] Z. Xiao, L. Ponnambalam, X. Fu, and W. Zhang, "Maritime traffic probabilistic forecasting based on vessels' waterway patterns and motion behaviors," IEEE Transactions on Intelligent Transportation Systems, vol. 18, no. 11, pp. 3122-3134, 2017.

[14] Y.-C. Chen, "A tutorial on kernel density estimation and recent advances," Biostatistics \& Epidemiology, vol. 1, no. 1, pp. 161-187, 2017. 
[15] B. Kawan, H. Wang, G. Li, and K. Chhantyal, "Data-driven modeling of ship motion prediction based on support vector regression," in Conference on Simulation and Modelling, 2017, pp. 350-354.

[16] H. Drucker, C. J. Burges, L. Kaufman, A. J. Smola, and V. Vapnik, "Support vector regression machines," in Advances in neural information processing systems 9, 1997, pp. 155-161.

[17] D. Nguyen, R. Vadaine, G. Hajduch, R. Garello, and R. Fablet, "A multi-task deep learning architecture for maritime surveillance using AIS data streams," in 2018 IEEE 5th International Conference on Data Science and Advanced Analytics (DSAA). IEEE, 2018, pp. 331-340.

[18] J. Chung, K. Kastner, L. Dinh, K. Goel, A. C. Courville, and Y. Bengio, "A recurrent latent variable model for sequential data," in Advances in neural information processing systems, 2015, pp. 2980-2988.

[19] D. Zissis, E. K. Xidias, and D. Lekkas, "Real-time vessel behavior prediction," Evolving Systems, vol. 7, no. 1, pp. 29-40, 2016.

[20] Marine cadastre vessel traffic data. [Online]. Available: https://marinecadastre. gov/ais/

[21] T. P. development team, "pandas-dev/pandas: Pandas," Feb. 2020. [Online]. Available: https://doi.org/10.5281/zenodo.3509134

[22] I. Goodfellow, Y. Bengio, and A. Courville, Deep Learning. MIT Press, Cambridge, 2016, http://www.deeplearningbook.org.

[23] D. P. Kingma and J. Ba, "Adam: A method for stochastic optimization," arXiv preprint arXiv:1412.6980, 2014. 
[24] F. Chollet et al. (2015) Keras. [Online]. Available: https://github.com/fchollet/ keras 